\title{
ON THE DYNAMIC DILATATION OF A COMPRESSIBLE RIVLIN CUBE BEYOND ITS ELASTIC LIMIT
}

\author{
KOSTAS P. SOLDATOS \\ School of Mathematical Sciences, University of Nottingham, Nottingham NG7 2RD, UK \\ and
}

Spencer Institute of Theoretical and Computational Mechanics, University of Nottingham, Nottingham, NG7

2RD, UK

\begin{abstract}
When an isotropic hyperelastic unit cube subjected to dynamic tri-axial extension/compression dilates successfully beyond its elastic limit, namely into its work-hardening deformation regime, plastic flow transforms any kind of induced into permanent anisotropy. If, for instance, two pairs of forces are identical while the third pair is different, then the initially isotropic material properties will transform permanently into those of transverse isotropy. For this problem, a plasticity model is presented that enables the energy stored during the work-hardening deformation stage of the resulting cuboid to be influenced not only by a tensorial measure of the observed deformation, but also by a measure of the plastic flow that takes place simultaneously. The model considers that plastic flow still obeys conventional plastic yield criteria, but does not postulate a-priory a rule that splits the observed deformation into elastic and plastic parts. Derivation of constitutive equations is based instead on the postulate that the strain energy density of the material is a function of the deformation gradient tensor and either the rate-of-plastic-deformation tensor encountered during loading within the work-hardening deformation regime or the residual strain tensor encountered after unloading is completed from some relevant offset yield point. An example application presents a complete analytical solution to the deformation problem of a dynamically loaded Rivlin cube which is made initially of a compressible Rivlin-Mooney material.
\end{abstract}

Keywords: Constitutive equations, Dilatation beyond elastic limit, Hyperelasticity, Induced anisotropy, Mooney-Rivlin material, Plasticity, Residual strain/stress, Rivlin's cube, Work-hardening plasticity, Yield condition.

\section{Introduction}

The classical problem of the Rivlin cube [1] refers to the stability and uniqueness/non-uniqueness of the dilatation states of an isotropic, incompressible hyperelastic unit cube which is acted upon by three identical pairs of equal and oppositely directed forces, $\pm f$, applied normally to its faces and distributed uniformly over them. The considerable number of relevant studies that followed [1] may be traced through a relatively smaller set of more recent publications [2-8], all of which dealt with stability and uniqueness/non-uniqueness of the dilatation states of a corresponding compressible hyperelastic cube.

This investigation aims to initiate a study of the dilatation states of an initially isotropic, hyperelastic and compressible unit cube which (i) develop in a dynamic manner, and (ii) reach and exceed the elastic limit of the cube material before any kind of possible material instability interferes with the deformation. Such dilatation states are expected to remain intact even after initial yield of the cube material takes place, namely within the work-hardening dilatation regime where elastic deformation and plastic flow take place simultaneously. 
Dilatation states of present interest are enforced on the cube through external action of appropriately developing dynamic, rather than dead loading conditions of the kind met in [1-8]. The aforementioned externally applied pairs of oppositely directed forces are still applied normally to the cube faces, over which they are still distributed uniformly, but are now not necessarily identical. They are, instead, expected to evolve through time in a manner that enables full control of the dilatation pattern of interest.

In some detail (see also Figure 1 and Section 2), complete unloading before the elastic limit is reached will naturally enable the material to recover both its initial cubical shape and initial stress state (see Figure 2(a)). However, due to residual strain accumulation that takes place within a work-hardening deformation regime, complete unloading from a state that lies beyond the elastic limit is expected to reshape the cube into the form of a residually strained cuboid (e.g., Figure 2(b) with $\hat{f}=f=0$ ). The shape of the resulting cuboid, as well as its dimensional and material differences from the initial, non-loaded cube are expected to relate closely to the influence that plastic flow has previously exerted on the material. Moreover, interference of the anticipated plastic flow will naturally transform any kind of induced anisotropy, caused previously in elastic deformation regime (e.g., [6]), into permanent material anisotropy.

Any induced anisotropy observed before initial yield is reached becomes thus part of the implied differences between the initial cubical and the final cuboidal shape of the deformable solid of interest. If, for instance, all three mutually perpendicular pairs of forces that cause dilatation of a compressible isotropic unit cube are unequal, then the degree of the permanent anisotropy observed within the work-hardening deformation regime will be that of an orthotropic material. If, on the other hand, only two of those pairs of forces are identical while the third pair of forces is different (e.g., Figure 2(b)), then the initial properties of material isotropy will transform permanently into those of transverse isotropy.

The outlined general considerations are thus valid regardless of the degree that the externally applied pairs of forces fail to be identical. However, the deformation gradient tensor, $\boldsymbol{F}$, attains a diagonal form, and retains that form throughout the implied deformation process. For the sake of simplicity though, the present study will develop around one of the simplest relevant problems. Namely (see Figure 2(b)), the problem in which $(i)$ like in [6], two of the externally applied pairs of equal and oppositely directed forces are identical ( $\pm f$ say), but different from the third pair, $\pm \hat{f}(\hat{f} \neq f)$; and (ii) those forces evolve in a manner that enables the cube to undergo quasi-static uniform extension without lateral contraction.

A proper description of this problem and the diagonal form attained by the deformation gradient tensor are provided in Section 2. That Section also describes in detail the fundamental features of the underpinning isothermal elastic and/or elastic-plastic material behaviour. In subsequent Sections, mathematical modelling always begins with a general, essentially arbitrary form of $\boldsymbol{F}$, thus implying that the model can handle successfully more general dilatation problems. Nevertheless, each Section finally focuses on the aforementioned pilot problem of uniform cube extension, with the aim of describing a complete analytical solution. The latter refers to the particular case in which the initial isotropic hyperelastic cube is made of a certain type of compressible Rivlin-Mooney material.

In this context, Section 3 provides details of the cube dilatation before initial yield is reached, while Section 4 describes the manner in which plastic flow is modelled during the work-hardening stage of loading. With the help of Appendix A, Section 5 then provides details of the appropriate constitutive modelling that combines the plastic flow and the elastic deformation processes observed during work-hardening. The previously induced properties of transverse isotropy are thus successfully incorporated into the permanent material properties of the evolving cuboid, while the resulting constitutive equations are found to be of a differential, rather than of a purely algebraic nature. Section 6 is devoted into modelling the manner in which unloading from some "offset yield point" attained within the work-hardening deformation regime enables the material to respond in a residually strained elastic manner. Prediction of the completely unloaded but, still, residually strained state that can finally be attained by the evolving cuboid is also among the aims of Section 6.

As is already mentioned, each of Sections 3-6 ends by providing details of a corresponding analytical solution achieved for the particular case that the initial isotropic hyperelastic cube is made of a certain type of 
compressible Rivlin-Mooney material. Cases in which the initial hyperelastic cube is made of some different isotropic material can be treated in a similar manner. This is one of several important conclusions detailed in Section 7, which makes also reference to possible future research directions.

\section{Problem description}

Consider a Cartesian coordinate system $\mathrm{O} x_{i}$ having each one of its axes normal to a pair of faces of a unit cube made of compressible material; here and in what follows Latin indices take values 1,2 and 3. At time $t$ $=t_{0}>0$, the cube is in an undeformed, unstrained and possibly unstressed state (Figure 2(a)), and has constant material density $\rho_{0}$. The position vector of a generic point of its elastic and isotropic material is denoted by $\boldsymbol{X}$ $=\left(X_{A}\right)$, where $\left|X_{A}\right| \leq 1 / 2$. At $t>t_{0}$, the cube is acted upon by a pair of equal and opposite forces, $\pm \hat{f}(t)$, applied normally to the faces which are normal to the $x_{1}$-axis, and two more pairs of equal and opposite forces, $\pm f(t)$, applied along the transverse co-ordinate directions, normal to the cube faces which are parallel to the $x_{1}$-axis (e.g., Figure 2(b)). Each of these forces is distributed uniformly over the corresponding cube face.

Under the assumption that $\hat{f} \neq f$, each pair of forces evolves in time in a prescribed manner, or in a manner that enables the corresponding cube edges to follow some prescribed dynamic deformation pattern that maintains cuboidal dilatation features. It is accordingly that the deformation gradient tensor, $\boldsymbol{F}$, obtains and throughout time retains a diagonal form, such that $F_{11} \neq F_{22}=F_{33}$. In this context, discussion necessarily focuses on possible cube dilatation patterns that take place before any kind of material instability is observed.

Here as well as in what follows, loading is generally associated with increasing values of $t>t_{0}$. Unloading generally takes place by decreasing the value of $t>t_{0}$ associated with some previously attained state of deformation. Rather than strictly representing time, $t$ is perceived as a parameter that accounts for a succession of deformation states.

The implied loading and deformation conditions are consistent with well-known fundamental features of isothermal hyperelastic and/or elastic-plastic material behaviour of solid continua. These features are detailed, for instance, in the introduction of a seminal relevant paper [9], as well as in most plasticity and other continuum mechanics textbooks (e.g., [10-12]). For completeness of this investigation, they are briefly described with the help of Figure 1 as follows:

(i) Response to increasing loading from the unstressed and unstrained configuration $\left(t=t_{0}\right)$ is and remains hyperelastic until an elastic limit point, $A$, that lies on the initial yield surface of the material is reached, at $t=t_{A} \geq t_{0}$ say. This initial hyperelastic stage of deformation is represented by a monotonically increasing load-strain curve (Figure 1).

(ii) With increasing loading beyond the recorded elastic limit, the material enters a work-hardening regime where, due to simultaneous action of elastic deformation and plastic flow, accumulation of increments of strain is enabled through application of progressively smaller increments of external loading. The loadstrain curve is still monotonically increasing but its slope is decreasing fast (Figure 1). Unloading cannot longer reverse the recorded yielding process.

(iii) Unloading from some "offset yield point" $B$ within the work-hardening regime (ii) enables the material to respond again hyperelastically by following a load-strain path which is generally different to the hyperelastic path $O A$ (Figure 1). Complete unloading brings the material to a residually strained state $C$. Unloading towards, or loading from such a residually strained state $C$ obeys again the rules (i)-(ii) above, with the initial yield point $A$ being now replaced by the offset yield point $B$. Hence, work-hardening beyond $B$ continues on the path shown in regime (ii) of the Figure 1.

This known idealised deformation process neglects a small hysteresis loop that may be observed after the unloading attempted from $B$ to $C$ is initially fully completed, and afterwards completely reversed (e.g. [1012]). An interplay of the outlined unloading-reloading process with possible small creeping phenomena may 
not be negligible. It is, however, considered as a higher-order effect and, hence, of secondary importance in this investigation, which is principally interested to propose a predictive-, rather than prescribed-type of plasticity constitutive formalism.

It is re-emphasised in this context that the present discussion refers only to cases in which the initial yield point $A$ of the cube material is reached before any kind of possible material instability, such as that discussed in [6], takes place. Initiation and, then, continuation of the work-hardening loading process within regime (ii) accumulates increasing amounts of residual strain. Unless that yielding process is ended through fracture or some other kind of material instability, many different unloading-loading elastic stages of the type (iii) may follow. In this context, any yield point $B$ of the loading path that represents stage (ii) in Figure 1, including the initial yield point $A$, lies on the material yield surface which may evolve with time.

These considerations can adequately be served by the assumption that the pair $\pm \hat{f}$ comprises either tensile or compressive forces and, hence, that $\hat{f}(t)>0$ or $\hat{f}(t)<0$, respectively, at all times. In dealing with the general description and analysis of the outlined problem, no other restrictions need to be imposed either on the sign and values of the applied forces $f$ and $\hat{f}$ or on the sign of their product $f \hat{f}$. The above criteria can be met by a large number of dilatation problems and an attempt is next made for the outlined theoretical analysis to be initially kept as general as possible.

Associated theoretical concepts are better understood when directly applied to some specific, pilot problem. The outlined general theoretical analysis will be built around the simple dynamic dilatation pattern

$x_{1}=X_{1} \frac{t}{t_{0}}, \quad x_{2}=X_{2}, \quad x_{3}=X_{3}, \quad\left(t \geq t_{0}\right)$.

This describes a quasi-static uniform extension without lateral contraction (Figure 2(b)), and becomes feasible only if the longitudinally applied forces that drive the deformation, namely $\pm \hat{f}$, are tensile $(\hat{f}(t)>0)$. The magnitude of this pair of forces and its evolution needs to be determined, along with the magnitude of the transverse counterparts, $\pm f(t)$. The latter may be perceived as pairs of follower or reaction forces that enable the transverse edges of the deforming cuboid to maintain their unit length.

The deformation gradient and the right Cauchy-Green deformation tensors associated with the dilatation pattern (2.1) attain the simple diagonal forms

$\boldsymbol{F}=\operatorname{diag}\left\{t / t_{0}, 1,1\right\}, \quad \boldsymbol{C}=\operatorname{diag}\left\{\left(t / t_{0}\right)^{2}, 1,1\right\}$,

respectively, and the corresponding velocity components, $v_{i}=d x_{i} / d t \equiv \dot{x}_{i}$, are given explicitly as follows:

$v_{1}=X_{1} / t_{0}=x_{1} / t, \quad v_{1}=v_{2}=0$,

where a dot denotes differentiation with respect to time. Hence, the corresponding rate-of-deformation tensor, $d_{i j}=\left(v_{i, j}+v_{j, i}\right) / 2$, involves only a single non-zero component, namely $d_{11}=1 / t$.

It is recalled that the mass of the continuum is required to obey the standard continuity equation

$\dot{\rho}+\rho v_{i, i}=0$,

where $\rho$ denotes the current mass density. In view of (2.1) and (2.3), (2.4) yields

$\rho=\rho_{0} t_{0} / t$

within the dilatation stages (i) and (ii).

It is also noted that the quasi-statically enforced deformation (2.1) is homogeneous and, hence, enables satisfaction of the relevant equations of motion,

$\sigma_{i j, i}=0$,

at least as long as the material remains isotropic; here, $\sigma$ represents the Cauchy stress tensor and body forces are neglected for simplicity. Nevertheless, the constitutive behaviour of the cube material needs to be resolved separately within each of the deformation regimes (i), (ii) and (iii).

It is emphasised that, regardless of whether the observed deformation is due to the particular dilatation pattern (2.1) or not, elastic deformation does take place in all three deformation regimes (i), (ii) and (iii). Within the work-hardening regime (ii), this is however evidently influenced by simultaneously occurring 
plastic flow while, within the elastic deformation regime (iii), it is similarly influenced by previously created residual strain. The strain energy density associated with the observed elastic deformation is expected to acquire different forms within each of those three different stages of material behaviour, and will be denoted $W^{I}, W^{I I}$ and $W^{I I I}$, respectively.

Appropriate continuity conditions should then hold at instances that two or possibly all three of those strain energy density branches merge. Those instances are represented in Figure 1 by the points $A$ and $B$, and enable the implied continuity conditions to be symbolically represented as follows:

$$
\left.W^{I}\right|_{A}=\left.W^{I I}\right|_{A},\left.\quad W^{I I}\right|_{B}=\left.W^{I I I}\right|_{B},\left.\quad W^{I I I}\right|_{B \equiv A}=\left.W^{I}\right|_{B \equiv A}=\left.W^{I I}\right|_{A} \text {. }
$$

An important feature of these continuity conditions is associated with the fact that the point $B$ represents a generic deformation state which is simultaneously part of the work-hardening stage (ii) and its purely elastic counterpart (iii).

Similar continuity conditions should also be satisfied at the merging points $A$ and $B$ by the stress states in the three deformation regimes. In complete analogy with (2.7), the implied stress continuity conditions are symbolically denoted as follows:

$$
\left.\boldsymbol{\sigma}^{I}\right|_{A}=\left.\boldsymbol{\sigma}^{I I}\right|_{A},\left.\quad \boldsymbol{\sigma}^{I I}\right|_{B}=\left.\boldsymbol{\sigma}^{I I I}\right|_{B},\left.\quad \boldsymbol{\sigma}^{I I I}\right|_{B \equiv A}=\left.\boldsymbol{\sigma}^{I}\right|_{B \equiv A}=\left.\boldsymbol{\sigma}^{I I}\right|_{B \equiv A}
$$

Relevant continuity conditions should finally also be satisfied at $A$ and $B$ by the total deformation encountered in the three regimes. Such conditions will be considered separately, wherever it is necessary and appropriate in what follows.

\section{The elastic deformation stage (i)}

\subsection{Constitution, stress and loading conditions}

In the hyperelastic deformation regime (i), where the strain energy density depends on the right CauchyGreen deformation tensor only,

$W^{I}=W^{I}(\boldsymbol{C})$,

material isotropy requires from $W^{I}$ to be a function of the principal deformation invariants

$$
I_{1}^{I}=\operatorname{tr} \boldsymbol{C}, \quad I_{2}^{I}=\frac{1}{2}\left[(\operatorname{tr} \boldsymbol{C})^{2}-\operatorname{tr} \boldsymbol{C}^{2}\right], I_{3}^{I}=\operatorname{det} \boldsymbol{C} .
$$

Following the standard procedure, one then obtains the well-known constitutive equation:

$$
\sigma_{i j}^{I}=\frac{\rho}{\rho_{0}} F_{i R} F_{j S}\left(\frac{\partial W^{I}}{\partial C_{R S}}+\frac{\partial W^{I}}{\partial C_{S R}}\right)=2 \frac{\rho}{\rho_{0}} F_{i R} F_{j S}\left\{\left(W_{1}^{I}+I_{1}^{I} W_{2}^{I}+I_{2}^{I} W_{3}^{I}\right) \delta_{R S}-\left(W_{2}^{I}+I_{1}^{I} W_{3}^{I}\right) C_{R S}+W_{3}^{I} C_{R P} C_{P S}\right\},
$$

where

$$
W_{\ell}^{I}=\frac{\partial W^{I}}{\partial I_{\ell}^{I}}, \quad(\ell=1,2,3) \text {. }
$$

This constitutive equation is valid for any finite elastic deformation, and its connection with the specific deformation (2.1) yields the non-zero stress components as follows:

$$
\begin{aligned}
& \sigma_{11}^{I}=2\left(t / t_{0}\right)\left(W_{1}^{I}+2 W_{2}^{I}+W_{3}^{I}\right), \\
& \sigma_{22}^{I}=\sigma_{33}^{I}=2\left(t_{0} / t\right)\left\{W_{1}^{I}+\left[\left(t / t_{0}\right)^{2}+1\right] W_{2}^{I}+\left(t / t_{0}\right)^{2} W_{3}^{I}\right\} .
\end{aligned}
$$

The external forces that maintain the dilatation (2.1) in stage (i) are

$\hat{f}=\sigma_{11}^{I} L_{0}^{2}=2\left(t / t_{0}\right) L_{0}^{2}\left(W_{1}^{I}+2 W_{2}^{I}+W_{3}^{I}\right)$,

$f=\sigma_{22}^{I} L L_{0}=\sigma_{22}^{I}\left(t / t_{0}\right) L_{0}^{2}=\sigma_{33}^{I}\left(t / t_{0}\right) L_{0}^{2}=2\left\{W_{1}^{I}+\left[\left(t / t_{0}\right)^{2}+1\right] W_{2}^{I}+\left(t / t_{0}\right)^{2} W_{3}^{I}\right\} L_{0}^{2}$,

where

$$
L=\left(t / t_{0}\right) L_{0}, \quad L_{0}=1
$$


denote length of the longitudinally oriented edges of the cuboid in the current and the reference configuration, respectively (Figure 2(b)).

It is observed that the forces (3.6) have initial values

$\left.\hat{f}\right|_{t=t_{0}}=\left.f\right|_{t=t_{0}}=f_{0}=2 L_{0}^{2}\left[W_{1}^{I}+2 W_{2}^{I}+W_{3}^{I}\right]_{t=t_{0}}$.

Unless

$\left[W_{1}^{I}+2 W_{2}^{I}+W_{3}^{I}\right]_{t=t_{0}}=0$,

the unit cube starts to experience the non-uniform dilatation (2.1) only after an appropriate level of pre-stress is reached. Strain energy densities expressible in the standard polynomial form, stemming from suitable truncation of the infinite power series proposed by Rivlin [13, 14], do indeed meet the initial condition (3.9), provided that their lowest degree terms are chosen to be at least quadratic in the strain invariants.

If the linear terms are retained in such a polynomial form of the strain energy density, then (3.9) may be violated and, as happens with the simple example application employed next in this Section, zero strain may not necessarily correspond to strictly zero external loading conditions. A possible violation of the principal postulates detailed in Section 2 is, however, still avoided by defining the loading parameter appearing in Figure 1 as follows:

$$
P=\max \left\{\left|\sigma_{11}^{I}-2\left[W_{1}^{I}+2 W_{2}^{I}+W_{3}^{I}\right]_{t=t_{0}}\right|,\left|\sigma_{22}^{I}-2\left[W_{1}^{I}+2 W_{2}^{I}+W_{3}^{I}\right]_{t=t_{0}}\right|\right\} .
$$

\subsection{Application: Compressible Mooney-Rivlin material}

The simplest possible form of a strain energy density of a compressible Mooney-Rivlin material, stemming from the afore-mentioned power-series truncation [13, 14], is as follows:

$$
W^{I}(\boldsymbol{C})=\frac{\mu_{1}}{2}\left(I_{1}^{I}-3\right)+\frac{\mu_{2}}{2}\left(I_{2}^{I}-3\right)+\mu_{3}\left(I_{3}^{I}-1\right)^{2} \text {, }
$$

where $\mu_{1}, \mu_{2}$ and $\mu_{3}$ are standard material moduli. It is noted that absence of a linear term in the third invariant enables equal amounts of volume change encountered under uniform compression and uniform extension to exert identical influence on $W^{I}$.

Expression (3.11) can be implemented in this example application without essential difficulty. However, the choice

$\mu_{3}=0$,

simplifies considerably formulas in subsequent Sections without invalidating any of the principal physical concepts of interest to this study. This choice of a value for $\mu_{3}$ reduces (3.11) into a form that resembles its counterpart associated with incompressible Mooney-Rivlin materials, but effects of material compressibility are still present as long as $I_{3}^{I} \neq 1$.

The non-zero stress components (3.5) then simplify to

$\sigma_{11}^{I}=\left(t / t_{0}\right)\left(\mu_{1}+2 \mu_{2}\right)$,

$\sigma_{22}^{I}=\sigma_{33}^{I}=\left(t_{0} / t\right)\left\{\mu_{1}+\left[\left(t / t_{0}\right)^{2}+1\right] \mu_{2}\right\}$,

and the external forces (3.6) that maintain the deformation (Figure 2(b)) are

$\hat{f}=\left(t / t_{0}\right) L_{0}^{2}\left(\mu_{1}+2 \mu_{2}\right)$,

$f=\left\{\mu_{1}+\left[\left(t / t_{0}\right)^{2}+1\right] \mu_{2}\right\} L_{0}^{2}$.

The unit cube can begin to experience the non-uniform dilatation (2.1) only after this set of forces enable the material to reach the pre-stress level

$\left.\sigma_{11}^{I}\right|_{t=t_{0}}=\left.\sigma_{22}^{I}\right|_{t=t_{0}}=\left.\sigma_{33}^{I}\right|_{t=t_{0}}=\mu_{1}+2 \mu_{2}$.

It is noted that this result holds regardless of the value of the material parameter $\mu_{3}$. Nevertheless, in the particular case that (3.12) holds, (3.10) provides the loading parameter appearing in Figure 1 as follows:

$P=\max \left\{\left(t / t_{0}-1\right)\left|\mu_{1}+2 \mu_{2}\right|,\left|\left(t_{0} / t-1\right) \mu_{1}+\left[\left(t / t_{0}\right)+\left(t_{0} / t\right)-2\right] \mu_{2}\right|\right\}$. 
The outlined results are valid until the transition instant, $t_{A}\left(\geq t_{0}\right)$, that separates the elastic dilatation stage (i) from its work-hardening counterpart (ii) is reached, i.e. until the driving force of the deformation and the longitudinal dimension of the deforming cuboid reach the values

$\hat{f}_{A}=\left(t_{A} / t_{0}\right)\left(\mu_{1}+2 \mu_{2}\right) L_{0}^{2}$,

$L_{A}=L_{0} t_{A} / t_{0}$.

However, the value of $t_{A}$ can be determined only after the plastic flow that emerges in region (ii) is properly accounted for.

\section{Plastic flow considerations}

Loading beyond the initial yield point level $A$ (Figure 1) forces the deformation into the work-hardening stage (ii) where dilatation is due to combined action of elastic strain and plastic flow. In accordance with basic concepts of the theory of plasticity, it is postulated that there exists a yield function $\varphi\left(\sigma_{i j}\right)$, such that $\varphi\left(\sigma_{i j}\right)-\kappa \leq 0$

for all admissible stress states, where $\kappa$ is a non-negative yield stress parameter.

If $\varphi<\kappa$, then the material responds elastically, as happens within the deformation regimes (i) and (iii) where $\sigma_{i j}=\sigma_{i j}^{I}$ and $\sigma_{i j}=\sigma_{i j}^{I I I}$, respectively. Equality in (4.1) holds only under conditions of loading within the work-hardening regime (ii), where $\sigma_{i j}=\sigma_{i j}^{I I}$ and the value of $\kappa$ is expected to increase or stay unaltered.

The non-negative parameter $\kappa$ is thus regarded as a non-decreasing function of some hardening measure, $q$, which characterises plastic deformation that took place already at any instant $t>t_{A}$ within stage (ii) (e.g., [12]). The required non-decreasing form of $\kappa(q)$ and its values beyond the initial value, $\kappa_{A}=\left.\kappa\right|_{t=t_{A}}$,

enable the yield function to expand with increasing loading, and, hence, to simulate satisfactorily the kind of proportional (often called "isotropic") hardening pattern observed in experiments.

The mathematical modelling approach adopted in the present analysis does not require a-priori specification of the parameter $q$ or the form of a function $\kappa(q)$. As is detailed in the example applications discussed later in Sections 4.3 and 5.2, these become known during the solution of particular problems.

\subsection{Development of the yield condition}

It is now recalled that $\varphi$ is required to be a function of the stresses through an appropriate, complete, and preferably irreducible basis of stress invariants. The number of the elements of such a basis depends on the material symmetries of the non-uniformly dilated cube. However, in the present case, the initial material symmetries of isotropy change into those of transverse isotropy as soon as plastic flow is initiated at $t=t_{A}$.

It is then understood that in the deformation regime (i) the yield condition (4.1) should be represented as follows:

$\varphi^{I}\left(J_{1}^{I}, J_{2}^{I}, J_{3}^{I}\right)-\kappa_{A}<0$, if $t<t_{A}$,

where

$J_{1}^{I}=\operatorname{tr} \sigma^{I}, \quad J_{2}^{I}=\operatorname{tr}\left(\sigma^{I}\right)^{2}, \quad J_{3}^{I}=\operatorname{tr}\left(\sigma^{I}\right)^{3}$,

are the principal stress invariants in the case of isotropy. However, in the deformation regime (ii), where plastic flow gives rise to permanent transverse isotropy along the direction of the unit vector $\boldsymbol{e}_{1}=(1,0,0)^{\mathrm{T}}$, the corresponding representation changes into the following:

$\varphi^{I I}\left(J_{1}^{I I}, J_{2}^{I I}, J_{3}^{I I}, J_{4}^{I I}, J_{5}^{I I}\right)-\kappa=0$, if $t \geq t_{A}$,

where, 
$J_{1}^{I I}=\operatorname{tr} \sigma^{I I}, J_{2}^{I I}=\operatorname{tr}\left(\sigma^{I I}\right)^{2}, J_{3}^{I I}=\operatorname{tr}\left(\sigma^{I I}\right)^{3}$,

$J_{4}^{I I}=\boldsymbol{e}_{1}^{T} \boldsymbol{\sigma}^{I I} \boldsymbol{e}_{1}=\sigma_{11}^{I I}, J_{5}^{I I}=\boldsymbol{e}_{1}^{T}\left(\boldsymbol{\sigma}^{I I}\right)^{2} \boldsymbol{e}_{1}=\left(\sigma_{11}^{I I}\right)^{2}=\left(J_{4}^{I I}\right)^{2}$.

It is observed that $J_{4}^{I I}$ and $J_{5}^{I I}$ are not independent in the present unidirectional cube dilation problem. For simplicity, direct involvement of $J_{5}^{I I}$ is thus hidden in what follows.

The fact that, at $t=t_{A}, \sigma_{i j}^{I}$ and (4.2) transform into $\sigma_{i j}^{I I}$ and (4.4), respectively, in a continuous manner, gives rise to the continuity conditions:

$$
\left.\sigma_{i j}^{I}\right|_{t=t_{A}}=\left.\sigma_{i j}^{I I}\right|_{t=t_{A}}, \lim _{t \rightarrow t_{A}} \varphi^{I}\left(J_{1}^{I}, J_{2}^{I}, J_{3}^{I}\right)=\left.\varphi^{I I}\left(J_{1}^{I I}, J_{2}^{I I}, J_{3}^{I I}, J_{4}^{I I}\right)\right|_{t=t_{A}}=\kappa_{A},
$$

the former of which is essentially an alternative representation of (2.8a). The corresponding deformation continuity condition associated with the pilot deformation pattern (2.1) is evidently satisfied by default.

\subsection{Plastic flow rule}

It is now postulated that the rate of the energy dissipation due to plastic flow,

$\psi=d_{i j}^{p} \sigma_{i j}^{I I}$,

is stationary; here $\boldsymbol{d}^{p}$ represents the rate-of-plastic-deformation tensor. This is adopted in classical plasticity (see page 51 of [10]), as well as in non-linear visco-plasticity [15] where the anticipated stationary point is expected to be a minimum. With the introduction of a Lagrange multiplier, $\lambda$, this postulate replaces (4.7) with the equivalent expression

$\psi=d_{i j}^{p} \sigma_{i j}^{I I}-\lambda\left[\varphi^{I I}\left(\sigma_{i j}^{I I}\right)-\kappa\right]$

whose minimisation leads to the well-known and relatively simple flow rule

$d_{i j}^{p}=\lambda \frac{\partial \varphi^{I I}}{\partial \sigma_{i j}^{I I}}$.

With use of (4.4) and (4.5), (4.9) leads to

$d_{i j}^{p}=\lambda\left(\varphi_{1}^{I I} \delta_{i j}+2 \varphi_{2}^{I I} \sigma_{i j}^{I I}+3 \varphi_{3}^{I I} \sigma_{i k}^{I I} \sigma_{k j}^{I I}+\varphi_{4}^{I I} \delta_{i 1} \delta_{j 1}\right)$,

where,

$\varphi_{\ell}^{I I}=\frac{\partial \phi^{I I}}{\partial J_{\ell}^{I I}}, \quad(\ell=1,2,3,4)$,

and, as already mentioned, (4.5e) justifies an apparent exclusion of $J_{5}^{I I}$. In case that Lagrangean rather than Eulerian description of the deformation is employed, (4.10) is replaced by its pull-back counterpart

$D_{R S}^{p}=F_{R i}^{-1} F_{S j}^{-1} d_{i j}^{p}=\lambda\left\{\varphi_{1}^{I I} C_{R S}^{-1}+F_{R i}^{-1} F_{S j}^{-1}\left(2 \varphi_{2}^{I I} \sigma_{i j}^{I I}+3 \varphi_{3}^{I I} \sigma_{i k}^{I I} \sigma_{k j}^{I I}\right)+F_{R 1}^{-1} F_{S 1}^{-1} \varphi_{4}^{I I}\right\}$.

Determination of the value of the Lagrange multiplier, $\lambda$, can become possible with the help of the continuity condition (4.6a) after the stress state is determined within the work-hardening stage (ii). The manner in which $\lambda$ is to be determined is demonstrated later, in Section 5.2, with an example application.

\subsection{Example: Quadratic branches of the yield function}

By tradition, metal plasticity employs yield functions which are quadratic in the stresses. In the present case, this tradition transforms the general yield function branches (4.2) and (4.4) into the following 


$$
\begin{aligned}
& \varphi^{I}\left(\sigma_{i j}^{I}\right)=J_{2}^{I} / 2=\frac{1}{2} \sigma_{i j}^{I} \sigma_{i j}^{I}=\frac{1}{2}\left[\left(\sigma_{11}^{I}\right)^{2}+\left(\sigma_{22}^{I}\right)^{2}+\left(\sigma_{33}^{I}\right)^{2}\right] \\
& \varphi^{I I}\left(\sigma_{i j}^{I I}\right)=\frac{1}{2}\left[J_{2}^{I I}+2 \alpha_{1} J_{1}^{I I} J_{4}^{I I}+\alpha_{2}\left(J_{4}^{I I}\right)^{2}\right]=\frac{1}{2}\left[\sigma_{i j}^{I I} \sigma_{i j}^{I I}+\sigma_{11}^{I I}\left(\alpha_{1} \sigma_{i j}^{I I} \delta_{i j}+\alpha_{2} \sigma_{11}^{I I}\right)\right]
\end{aligned}
$$

The coefficients $\alpha_{1}$ and $\alpha_{2}$ may initially be regarded as additional material moduli. However, full consideration of the continuity condition (4.6b), with simultaneous use of (4.6a), leads to the relationship

$$
\alpha_{2}=-\alpha_{1}\left(1+\left.2 \frac{\sigma_{22}^{I}}{\sigma_{11}^{I}}\right|_{t=t_{A}}\right)
$$

so that the parameters $\alpha_{1}$ and $\alpha_{2}$ are not independent (see also (4.17) and (4.20) below).

Connection of (4.10) and (4.12) with (4.13b) provides the flow rule in the following dual form:

$d_{i j}^{p}=\lambda\left\{\sigma_{i j}^{I I}+\sigma_{11}^{I I}\left[\alpha_{1} \delta_{i j}+\left(\alpha_{1}+\alpha_{2}\right) \delta_{i 1} \delta_{j 1}\right]+\alpha_{1}\left(\sigma_{22}^{I I}+\sigma_{33}^{I I}\right) \delta_{i 1} \delta_{j 1}\right\}$,

$D_{R S}^{p}=\lambda F_{R i}^{-1} F_{S j}^{-1}\left\{\sigma_{i j}^{I I}+\sigma_{11}^{I I}\left[\alpha_{1} \delta_{i j}+\left(\alpha_{1}+\alpha_{2}\right) \delta_{i 1} \delta_{j 1}\right]+\alpha_{1}\left(\sigma_{22}^{I I}+\sigma_{33}^{I I}\right) \delta_{i 1} \delta_{j 1}\right\}$,

where (4.11) enabled use of the results $\varphi_{1}^{I I}=\alpha_{1} J_{4}^{I I}, \varphi_{2}^{I I}=1 / 2, \varphi_{3}^{I I}=0$ and $\varphi_{4}^{I I}=2 \alpha_{1} J_{1}^{I I}+2 \alpha_{2} J_{4}^{I I}$.

The outlined observations become better understood in the particular case of the pilot deformation (2.1). Accordingly, upon inserting constitutive equation (3.5) into (4.13a) and making use of the first branch of the continuity conditions (4.6b), one finds that $t_{A}\left(\geq t_{0}\right)$ should be sought from the positive real roots of the algebraic equation

$2\left[\left(t / t_{0}\right)^{2}\left(W_{1}^{I}+2 W_{2}^{I}+W_{3}^{I}\right)^{2}+2\left(t_{0} / t\right)^{2}\left\{W_{1}^{I}+\left(\left(t / t_{0}\right)^{2}+1\right) W_{2}^{I}+\left(t / t_{0}\right)^{2} W_{3}^{I}\right\}^{2}\right\rfloor_{t=t_{A}}-\kappa_{A}=0$.

Hence, use of (4.15) yields the following relationship between the newly introduced parameters:

$\alpha_{2}=-\alpha_{1}\left\{1+2\left[\frac{\left(W_{1}^{I}+W_{2}^{I}\right)\left(t_{0} / t_{A}\right)^{2}+W_{2}^{I}+W_{3}^{I}}{W_{1}^{I}+2 W_{2}^{I}+W_{3}^{I}}\right]_{t=t_{A}}\right\}$.

Another equation, which involves partial derivatives of $W^{I I}$ and may be considered as a second relationship between $\alpha_{1}$ and $\alpha_{2}$, will emerge later in Section 6.4, and with (4.17) are considered a pair of simultaneous algebraic equations for $\alpha_{1}$ and $\alpha_{2}$. This observation will make thus more generally understood that $\alpha_{1}$ and $\alpha_{2}$ are not new material parameters, but they both depend on their $W^{I}$ and $W^{I I}$ counterparts. Nevertheless, the specific manner in which $t_{A}$ enters (4.16) and (4.17) suggests further that the values of $\alpha_{1}$ and $\alpha_{2}$ may depend not only on the chosen form of the strain energy density, but also on the manner in which time-depended dilatation is imposed on the deforming cuboid.

\subsection{Application: Compressible Mooney-Rivlin material}

In the particular case of the Mooney-Rivlin material considered in Section 3.2, (3.11) and (3.12) enable (4.16) to obtain the form of a biquadratic equation, namely

$2\left[\left(\mu_{1}+2 \mu_{2}\right)^{2}+2 \mu_{2}^{2}\right]\left(t_{A} / t_{0}\right)^{4}+\left[8 \mu_{2}\left(\mu_{1}+\mu_{2}\right)-\kappa_{A}\right]\left(t_{A} / t_{0}\right)^{2}+4\left(\mu_{1}+\mu_{2}\right)^{2}=0$.

It can be shown that real solutions of this equation are possible only if

$\kappa_{A} \geq \sqrt{1+2\left(1+\mu_{1} / 2 \mu_{2}\right)^{2}}$.

Provided that this inequality is satisfied, then admissible values of $t_{A}\left(\geq t_{0}\right)$ should be sought among the positive real roots of (4.18).

Moreover, (4.14) or, equivalently, (4.17) reduces to

$$
\alpha_{2}=-\alpha_{1}\left(1+2 \frac{\left(\mu_{1}+\mu_{2}\right)\left(t_{0} / t_{A}\right)^{2}+\mu_{2}}{\mu_{1}+2 \mu_{2}}\right) \text {. }
$$

This shows that the values of $\alpha_{1}$ and $\alpha_{2}$ are interrelated, and are also dependent on the values of the material moduli involved in the hyperelastic deformation stage (i) of the material. Nevertheless, the more general observations detailed earlier (see last paragraph of Section 4.3) still apply in this particular case. 
In the pilot case of present interest, where $\boldsymbol{F}$ is given by (2.2), the non-zero components of the rateof-plastic-deformation tensors (4.17) are found to be $\left(t>t_{A}\right)$

$$
\begin{aligned}
& d_{11}^{p}=\lambda\left\{\left(1+2 \alpha_{1}+\alpha_{2}\right) \sigma_{11}^{I I}+2 \alpha_{1} \sigma_{22}^{I I}\right\}, \\
& D_{11}^{p}=\lambda\left(t_{0} / t\right)^{2}\left\{\left(1+2 \alpha_{1}+\alpha_{2}\right) \sigma_{11}^{I I}+2 \alpha_{1} \sigma_{22}^{I I}\right\}, \\
& D_{22}^{p}=D_{33}^{p}=d_{22}^{p}=d_{33}^{p}=\lambda\left(\sigma_{22}^{I I}+\alpha_{1} \sigma_{11}^{I I}\right) .
\end{aligned}
$$

Here, (4.20) still holds, while use is also made of the expectation that

$\sigma_{33}=\sigma_{22}$

(4.22)

in all three deformation stages (i), (ii) and (iii); see also (3.5).

It is emphasised that a constitutive equation that provides the stress components appearing in (4.21) is currently still unavailable. This will be sought and determined next in Section 5, with the help of a more general relevant development detailed in Appendix A. After inserted into (4.13b), the stress components sought will enable (4.4) to take the form of the aforementioned rule, $\kappa(q)$, that describes the expansion of the yield surface within the work-hardening deformation stage. The currently unknown hardening parameter, $q$, will naturally be involved in that expression, and will thus reveal itself at the same time.

\section{The work-hardening deformation stage (ii)}

During work-hardening, plastic flow transforms the direction $\boldsymbol{e}_{1}=(1,0,0)^{\mathrm{T}}$ into a direction of permanent transverse isotropy. Relevant constitutive equations in the deformation stage (ii) are sought by initially replacing the unit vector $\boldsymbol{A}$ appearing in (A.11), (A.12a) and (A.16) with $\boldsymbol{e}_{1}$, thus leading to

$$
\begin{aligned}
\sigma_{i j}^{I I}=2 \frac{\rho}{\rho_{0}} F_{i R} F_{j S}\left\{\left(W_{1}^{I I}+I_{1}^{I I} W_{2}^{I I}\right.\right. & \left.\left.+I_{2}^{I I} W_{3}^{I I}\right) \delta_{R S}-\left(W_{2}^{I I}+I_{1}^{I I} W_{3}^{I I}\right) C_{R S}+W_{3}^{I I} C_{R P} C_{P S}\right\} \\
& +2 \frac{\rho}{\rho_{0}}\left\{F_{i 1} F_{j 1} W_{4}^{I I}+W_{5}^{I I}\left(F_{i 1} F_{j S} C_{S 1}+F_{i R} F_{j 1} C_{R 1}\right)\right\}+\Theta_{i j}^{I I}(\boldsymbol{\sigma})+\Phi_{i j}^{I I}(\boldsymbol{\sigma}),
\end{aligned}
$$

where

$$
\begin{aligned}
& \Theta_{i j}^{I I}=2 \frac{\rho}{\rho_{0}} F_{i R} F_{j S}\left\{W_{6}^{I I} D_{R S}^{p}+W_{7}^{I I} D_{R P}^{p} D_{P S}^{p}+2\left(W_{8}^{I I} D_{R P}^{p}+W_{9}^{I I} D_{R Q}^{p} D_{Q P}^{p}\right) C_{P S}\right\}+2 \frac{\rho}{\rho_{0}} F_{i 1} F_{j S} W_{10}^{I I} D_{S 1}^{p}, \\
& \Phi_{i j}^{I I}=2 \frac{\rho}{\rho_{0}}\left(F_{i R} F_{j S} / \dot{C}_{R S}\right)\left(\dot{D}_{M N}^{p} E_{N M}\right),
\end{aligned}
$$

and

$$
\begin{aligned}
\dot{D}_{M N}^{p} E_{N M} & =\dot{D}_{1 N}^{p}\left(W_{10}^{I I} C_{N 1}+2 W_{15}^{I I} D_{N 1}^{p}\right)+\dot{D}_{M M}^{p} W_{11}^{I I}+\dot{D}_{11}^{p} W_{14}^{I I}+ \\
& +\dot{D}_{M N}^{p}\left\{W_{6}^{I I} C_{N M}+\left(2 W_{7}^{I I} D_{N P}^{p}+W_{8}^{I I} C_{N P}+2 W_{9}^{I I} C_{P Q} D_{Q N}^{p}\right) C_{M P}+2 W_{12}^{I I} D_{M N}^{p}+3 W_{13}^{I I} D_{M P}^{p} D_{P N}^{p}\right\} .
\end{aligned}
$$

This constitutive equation is valid regardless of the form of the imposed deformation but, in the present case of interest, the deformation pattern (2.1) simplifies the analysis considerably.

Use of the particular deformation characteristics detailed in (2.2) and (2.5) leads to

$\dot{\boldsymbol{C}}=\operatorname{diag}\left\{2 t / t_{0}^{2}, 0,0\right\}$,

and enables the deformation invariants (A.8) to simplify as follows: 
$I_{1}^{I I}=2+\left(t / t_{0}\right)^{2}, I_{2}^{I I}=1+2\left(t / t_{0}\right)^{2}, I_{3}^{I I}=\left(t / t_{0}\right)^{2}, I_{4}^{I I}=\boldsymbol{e}_{1}^{T} \boldsymbol{C} \boldsymbol{e}_{1}=C_{11}=\left(t / t_{0}\right)^{2}$,

$I_{5}^{I I}=\boldsymbol{e}_{1}^{T} \boldsymbol{C}^{2} \boldsymbol{e}_{1}^{T}=\left(t / t_{0}\right)^{4}, I_{6}^{I I}=\left(t / t_{0}\right)^{2} D_{11}^{p}+2 D_{22}^{p}, I_{7}^{I I}=\left(t / t_{0}\right)^{2}\left(D_{11}^{p}\right)^{2}+2\left(D_{22}^{p}\right)^{2}$,

$I_{8}^{I I}=\left(t / t_{0}\right)^{4} D_{11}^{p}+2 D_{22}^{p}, I_{9}^{I I}=\left(t / t_{0}\right)^{4}\left(D_{11}^{p}\right)^{2}+2\left(D_{22}^{p}\right)^{2}, I_{10}^{I I}=\boldsymbol{e}_{1}^{T} \boldsymbol{C} \boldsymbol{D}^{p} \boldsymbol{e}_{1}=\left(t / t_{0}\right)^{2} D_{11}^{p}$,

$I_{11}^{I I}=D_{11}^{p}+2 D_{22}^{p}, I_{12}^{I I}=D_{11}^{p^{2}}+2 D_{22}^{2}, I_{13}^{I I}=\left(D_{11}^{p}\right)^{3}+2\left(D_{22}^{p}\right)^{3}, I_{14}^{I I}=\boldsymbol{e}_{1}^{T} D^{p} \boldsymbol{e}_{1}=D_{11}^{p}$,

$I_{15}^{I I}=\boldsymbol{e}_{1}^{\boldsymbol{T}}\left(\boldsymbol{D}^{p}\right)^{2} \boldsymbol{e}_{1}=\left(D_{11}^{p}\right)^{2}$.

A specific choice of the yield function can similarly facilitate analytical progress by allowing the rate-ofplastic-deformation tensor to attain a corresponding specific form.

\subsection{Association of the dilatation pattern (2.1) with a quadratic yield function}

Association of (2.2) with the quadratic form (4.13b) of $\varphi^{I I}$ thus enables direct use of the non-zero components (4.21) of the rate-of-plastic-deformation tensor. Corresponding non-zero components of $\dot{\boldsymbol{D}}^{p}$ are then found to be

$$
\begin{aligned}
& \dot{D}_{11}^{p}=\lambda\left(t_{0} / t\right)^{2}\left[\left(1+2 \alpha_{1}+\alpha_{2}\right)\left(\dot{\sigma}_{11}^{I I}-2 \sigma_{11}^{I I} / t\right)+2 \alpha_{1}\left(\dot{\sigma}_{22}^{I I}-\sigma_{22}^{I I} / t\right)\right], \\
& \dot{D}_{22}^{p}=\dot{D}_{33}^{p}=\lambda\left(\dot{\sigma}_{22}^{I I}+\alpha_{1} \dot{\sigma}_{11}^{I I}\right),
\end{aligned}
$$

where (4.17) still holds and use is made of (4.22). The diagonal form of all tensors involved in the analysis thus makes it evident that the constitutive equation sought in the work-hardening regime becomes available as soon as the corresponding normal stress components are fully determined.

Consideration of (5.1) and (5.2b) reveals that determination of $\sigma_{22}^{I I}$ or, equivalently, $\sigma_{33}^{I I}$ implicates into the analysis the factor $1 / \dot{C}_{22}$ or, respectively, $1 / \dot{C}_{33}$ which is singular in the present case; see (5.4). However, either of the observed singularities is removed with use of the self-evident relations

$$
\lim _{\dot{C}_{22} \rightarrow 0}\left(\dot{C}_{22} \sigma_{22}^{I I}\right)=\lim _{\dot{C}_{33} \rightarrow 0}\left(\dot{C}_{33} \sigma_{33}^{I I}\right)=0 \text {. }
$$

When connected with (5.1), these lead to

$$
\lim _{\dot{C}_{22} \rightarrow 0}\left(\dot{C}_{22} \Phi_{22}^{I I}\right)=\lim _{\dot{C}_{33} \rightarrow 0}\left(\dot{C}_{33} \Phi_{33}^{I I}\right)=\dot{D}_{M N}^{p} E_{N M}=0 \text {, }
$$

and, by virtue of (5.3), to

$$
\begin{aligned}
& \dot{D}_{1 N}^{p}\left(W_{10}^{I I} C_{N 1}+2 W_{15}^{I I} D_{N 1}^{p}\right)+\dot{D}_{M M}^{p} W_{11}^{I I}+\dot{D}_{11}^{p} W_{14}^{I I}+ \\
& \quad+\dot{D}_{M N}^{p}\left\{W_{6}^{I I} C_{M N}+\left(2 W_{7}^{I I} D_{N P}^{p}+W_{8}^{I I} C_{P N}+2 W_{9}^{I I} C_{P Q} D_{Q N}^{p}\right) C_{M P}+2 W_{12}^{I I} D_{N M}^{p}+3 W_{13}^{I I} D_{M P}^{p} D_{P N}^{p}\right\}=0 .
\end{aligned}
$$

The quantity $\Phi_{22}^{I I}=\Phi_{33}^{I I}$ remains thus undetermined but further use of (4.21) and (5.6) can transform (5.9) into a differential constitutive equation for the unknown normal stress components. The manner in which this becomes possible is demonstrated with a particular example application in Section 5.2 below.

When combined with (5.8), (5.2b) reveals that $\Phi_{11}^{I I}=0$. Hence, (5.1) provides the longitudinal normal stress component in the following form:

$$
\sigma_{11}^{I I}=2\left(t / t_{0}\right)\left(W_{1}^{I I}+2 W_{2}^{I I}+W_{3}^{I I}+W_{4}^{I I}+W_{5}^{I I}\right)+\Theta_{11}^{I I}(\boldsymbol{\sigma}) \text {, }
$$

where,

$$
\Theta_{11}^{I I}=2\left(t / t_{0}\right)\left\{W_{6}^{I I}+W_{7}^{I I} D_{11}^{p}+W_{10}^{I I}+2\left(t / t_{0}\right)^{2}\left(W_{8}^{I I}+W_{9}^{I I} D_{11}^{p}\right)\right\}
$$

Connected with (4.21b), (5.11) makes clear that, unlike (5.9), (5.10) emerges in the form of a purely algebraic constitutive equation which, however, is still implicit in the unknown normal stress components. For further development, the set of constitutive equations (5.9) and (5.10) requires specification of the form of $W^{I I}$.

\subsection{Application: Work-hardening and fracture of a compressible Mooney-Rivlin cuboid}


Consider a compressible Mooney-Rivlin material which, in the work-hardening regime, is represented by the following extension of the strain energy density employed in Section 3.2:

$$
W^{I I}(\boldsymbol{C})=\frac{\mu_{1}}{2}\left(I_{1}^{I I}-3\right)+\frac{\mu_{2}}{2}\left(I_{2}^{I I}-3\right)+\frac{\tilde{\mu}_{1}}{2}\left(I_{4}^{I I}-\left.I_{4}^{I I}\right|_{t=t_{A}}\right)+\frac{\tilde{\mu}_{2}}{2}\left(I_{6}^{I I}-\left.I_{6}^{I I}\right|_{t=t_{A}}\right),
$$

where $\tilde{\mu}_{1}$ and $\tilde{\mu}_{2}$ are suitable material moduli, additional to those appearing in (3.11). It is noted that the adopted choices of $W^{I I}$ and $W^{I}$ do satisfy the strain energy density continuity condition (2.7a).

In the case of uniaxial deformation pattern (2.1), the form (5.5) of the deformation invariants enables (5.12) to convert into the following:

$$
W^{I I}(\boldsymbol{C})=\left(\frac{\mu_{1}}{2}+\mu_{2}\right)\left[\left(t / t_{0}\right)^{2}-1\right]+\frac{\tilde{\mu}_{1}}{2}\left[\left(t / t_{0}\right)^{2}-\left(t / t_{A}\right)^{2}\right]+\frac{\tilde{\mu}_{2}}{2}\left\{\left(t / t_{0}\right)^{2} D_{11}^{p}+2 D_{22}^{p}-\left.I_{6}^{I I}\right|_{t=t_{A}}\right\}, t \geq t_{A},
$$

where the appearing components of the rate-of-plastic-deformation tensor are generally given according to (4.12).

In the particular case of the quadratic yield function (4.13), (4.12) reduces to $(4.21 \mathrm{~b}, \mathrm{c})$ and, hence, (5.6) hold in association with (4.20). It is also seen that

$$
\begin{aligned}
\left.I_{6}^{I I}\right|_{t=t_{A}} & =\lambda\left[\left(1+4 \alpha_{1}+\alpha_{2}\right) \sigma_{11}^{I I}+2\left(1+\alpha_{1}\right) \sigma_{22}^{I I}\right]_{t=t_{A}} \\
& =2 \lambda\left[\left(t_{A} / t_{0}\right)\left(\mu_{1}+2 \mu_{2}\right)\left(1+4 \alpha_{1}+\alpha_{2}\right)+2\left(1+\alpha_{1}\right)\left(t_{0} / t_{A}\right)\left\{\mu_{1}+\left[\left(t_{A} / t_{0}\right)+1\right] \mu_{2}\right\}\right],
\end{aligned}
$$

where a combination of the stress continuity conditions (2.8a) with (3.13) also enables the use of

$$
\begin{aligned}
& \left.\sigma_{11}^{I I}\right|_{t=t_{A}}=\left.\sigma_{11}^{I}\right|_{t=t_{A}}=\left(t_{A} / t_{0}\right)\left(\mu_{1}+2 \mu_{2}\right), \\
& \left.\sigma_{22}^{I I}\right|_{t=t_{A}}=\left.\sigma_{22}^{I}\right|_{t=t_{A}}=\left(t_{0} / t_{A}\right)\left\{\mu_{1}+\left[\left(t / t_{A}\right)^{2}+1\right] \mu_{2}\right\} .
\end{aligned}
$$

The constitutive equation (5.8) or, equivalently, (5.9) then reduces to

$\dot{D}_{11}^{p} C_{11}+2 \dot{D}_{22}^{p} C_{22}=\lambda\left[\left(1+2 \alpha_{1}+\alpha_{2}\right)\left(\dot{\sigma}_{11}^{I I}-2 \sigma_{11}^{I I} / t\right)+2 \alpha_{1}\left(\dot{\sigma}_{22}^{I I}-\sigma_{22}^{I I} / t\right)\right]+2 \lambda\left(\dot{\sigma}_{22}^{I I}+\alpha_{1} \dot{\sigma}_{11}^{I I}\right)=0$,

which can be rearranged into the following form of a differential equation:

$$
\left(1+4 \alpha_{1}+\alpha_{2}\right) \dot{\sigma}_{11}^{I I}+2\left(1+\alpha_{1}\right) \dot{\sigma}_{22}^{I I}-(2 / t)\left[\left(1+2 \alpha_{1}+\alpha_{2}\right) \sigma_{11}^{I I}-\alpha_{1} \sigma_{22}^{I I}\right\rfloor=0 \text {. }
$$

In a similar manner, the constitutive equation (5.10) takes initially the form

$$
\sigma_{11}^{I I}\left[1-\lambda\left(t_{0} / t\right) \tilde{\mu}_{2}\left(1+2 \alpha_{1}+\alpha_{2}\right)\right]=\left(t / t_{0}\right)\left(\mu_{1}+2 \mu_{2}+\tilde{\mu}_{1}\right)+2 \lambda \alpha_{1} \tilde{\mu}_{2}\left(t_{0} / t\right) \sigma_{22}^{I I} \text {. }
$$

This can further simplify into the following:

$$
\sigma_{22}^{I I}=\sigma_{33}^{I I}=\sigma_{11}^{I I}\left[\lambda^{-1} \beta_{1}\left(t / t_{0}\right)-\beta_{2}\right]-\lambda^{-1} \beta_{3}\left(t_{0} / t\right)^{2},
$$

where

$$
\beta_{1}=\left(2 \alpha_{1} \tilde{\mu}_{2}\right)^{-1}, \quad \beta_{2}=\frac{\left(1+2 \alpha_{1}+\alpha_{2}\right)}{2 \alpha_{1}}, \beta_{3}=\frac{\mu_{1}+2 \mu_{2}+\tilde{\mu}_{1}}{2 \alpha_{1} \tilde{\mu}_{2}},
$$

and (4.22) is also accounted for. Subsequent combination of (5.15) with the value of (5.18) at $t=t_{A}$ yields the Lagrange multiplier $\lambda$ as

$$
\lambda=\frac{\left(t_{A} / t_{0}\right)^{2}\left(\mu_{1}+2 \mu_{2}\right)\left[\beta_{1}\left(t_{A} / t_{0}\right)-\beta_{2}\right]-\beta_{3}\left(t_{0} / t_{A}\right)}{\mu_{1}+\left[\left(t_{A} / t_{0}\right)^{2}+1\right] \mu_{2}} .
$$

Explicit constitutive equations, equivalent to their implicit counterparts (5.17) and (5.18), are next obtained, by initially differentiating (5.18) and, hence, arriving at the intermediate result $\dot{\sigma}_{22}^{I I}=\dot{\sigma}_{11}^{I I}\left[\lambda^{-1} \beta_{1}\left(t / t_{0}\right)-\beta_{2}\right]+\sigma_{11}^{I I} \lambda^{-1} \beta_{1} / t_{0}+2 \lambda^{-1} \beta_{3}\left(t_{0}^{2} / t^{3}\right)$.

Appropriate combination of (5.18) and (5.21), thus enables (5.16) to obtain the following form of a first order linear ordinary differential equation:

$\dot{\sigma}_{11}^{I I}+p(t) \sigma_{11}^{I I}=q(t)$

where 


$$
\begin{aligned}
& p(t)=\frac{2 \beta_{1}\left(t / t_{0}\right)-2 \lambda\left[1+\alpha_{1}\left(2-\beta_{2}\right)+\alpha_{2}\right]}{t\left\{2 \alpha_{1} \beta_{1}\left(t / t_{0}\right)+\lambda\left[1+2 \alpha_{1}\left(2-\beta_{2}\right)+\alpha_{2}\right]\right\}}, \\
& q(t)=\frac{-2 \beta_{3}\left(1+\alpha_{1}\right)\left(t_{0} / t\right)^{2}}{t\left\{2 \alpha_{1} \beta_{1}\left(t / t_{0}\right)+\lambda\left[1+2 \alpha_{1}\left(2-\beta_{2}\right)+\alpha_{2}\right]\right\}} .
\end{aligned}
$$

When subjected to the initial condition (5.15a), the general solution of (5.22) yields explicitly the longitudinal normal stress component in the form

$$
\sigma_{11}^{I I}=\frac{1}{K(t)}\left\{\left(t_{A} / t_{0}\right)\left(\mu_{1}+2 \mu_{2}\right) K\left(t_{A}\right)+\int_{t_{A}}^{t} K(t) q(t) d t\right\}, K(t)=\exp \left(\int p(t) d t\right) .
$$

Corresponding closed forms of the transverse normal stresses are finally obtained in a straightforward manner, by directly substituting (5.23) into (5.18). The stress components thus obtained are still functions of time only and, hence, satisfy the quasi-static equations of motion (2.6) identically.

It is noted with interest that the presented closed form solution of (5.22), namely (5.23), is exact for the stresses. However, some of the integrations noted in (5.23) have to be performed numerically, and this can become possible in practical applications only after numerical values are specified for the material parameters. The analytical part of the outlined solution can however still develop by initially observing that the external forces that maintain the assumed deformation within the work-hardening stage (Figure 2(b)) are obtained by inserting (5.23) into the right hand sides of

$$
\begin{aligned}
& \hat{f}=\sigma_{11}^{I I} L_{0}^{2}, \\
& f=\sigma_{22}^{I I}\left(t / t_{0}\right) L_{0}^{2}=\sigma_{33}^{I I}\left(t / t_{0}\right) L_{0}^{2}=\left(t / t_{0}\right)\left\{\sigma_{11}^{I I}\left[\lambda^{-1} \beta_{1}\left(t / t_{0}\right)-\beta_{2}\right]-\lambda^{-1} \beta_{3}\left(t_{0} / t\right)^{2}\right\} L_{0}^{2} .
\end{aligned}
$$

Moreover, by inserting (5.18) and (5.23) into (4.13b) and, then, combining the obtained result with the yield condition (4.4), one obtains the expansion rule of the yield surface in the following form:

$$
\kappa\left(\sigma_{11}^{I I}\right)=\frac{1}{2}\left[\left(1+\alpha_{1}+\alpha_{2}\right) \sigma_{11}^{I I} \sigma_{11}^{I I}+2 \sigma_{22}^{I I}\left(\sigma_{22}^{I I}+\alpha_{1} \sigma_{11}^{I I}\right)\right] \text {. }
$$

In view of (5.18) or (5.24a), (5.25) thus reveals that $\sigma_{11}^{I I}$ or $\hat{f}$, respectively, represents the aforementioned hardening measure, $q$, that characterises plastic deformation that took place at any $t>t_{A}$ in stage (ii) [12].

Determination of the non-zero components of the plastic flow tensor now becomes possible through direct use of (4.21). Time integration of the resulting forms of $d_{11}^{p}$ and $d_{22}^{p}$ yields thus the plastic deformation during work-hardening of the undeformed $\left(0<X_{A} \leq 1 / 2\right)$-part of the cube as follows:

$$
\begin{aligned}
& x_{1}^{p}=\lambda\left[\left(1+2 \alpha_{1}+\alpha_{2}\right) \int_{t_{A}}^{t} \sigma_{11}^{I I} d t+2 \alpha_{1} \int_{t_{A}}^{t} \sigma_{22}^{I I} d t\right], \\
& x_{2}^{p}=x_{3}^{p}=\lambda\left(\int_{t_{A}}^{t} \sigma_{22}^{I I} d t+\alpha_{1} \int_{t_{A}}^{t} \sigma_{11}^{I I} d t\right) .
\end{aligned}
$$

The elastic deformation in that part of the work-hardening material of the cube, namely

$$
\begin{aligned}
& x_{1}^{e}=X_{1}\left(t / t_{0}\right)-\lambda\left[\left(1+2 \alpha_{1}+\alpha_{2}\right) \int_{t_{A}}^{t} \sigma_{11}^{I I} d t+2 \alpha_{1} \int_{t_{A}}^{t} \sigma_{22}^{I I} d t\right]>0, \\
& x_{2}^{e}=x_{3}^{e}=X_{2}-\lambda\left(\int_{t_{A}}^{t} \sigma_{22}^{I I} d t+\alpha_{1} \int_{t_{A}}^{t} \sigma_{11}^{I I} d t\right)>0,
\end{aligned}
$$

is finally obtained by subtracting (5.26) from the total deformation (2.1). The discussed symmetries of the problem make evident the manner in which (5.26) and (5.27) can be generalised and, after suitable modification, produce their counterparts within the remaining three parts of the deforming cuboid.

Apart from obeying the relationship (4.14), the material parameters $\alpha_{1}$ and $\alpha_{2}$ should also be such that both inequalities (5.27) hold simultaneously within a time interval $\left[t_{A}, t_{F}\right)$, where $t_{F} \geq t_{A}$ represents the 
time instant that fracture takes place. That instant is thus specified as the first instant of time within the workhardening deformation stage (ii) at which

$\min \left(x_{1}^{e}, x_{2}^{e}=x_{3}^{e}\right)=0$,

for some $0<X_{A} \leq 1 / 2$. A search for the range and/or combination of the values of $\alpha_{1}$ and $\alpha_{2}$ that fulfil the outlined conditions requires a substantial amount of numerical work, which is however beyond the principal aims and objectives of this investigation.

\section{The residually strained elastic deformation stage (iii)}

Unloading from any yield point $B\left(t_{B}>t_{A}\right)$ within the work-hardening stage (ii) forces the deformation to follow some different stress-strain path on which the material again behaves elastically. Continuation or partial reversal of the implied unloading process leaves unaltered the observed material response, which remains elastic and, therefore, completely reversible within that newly developing deformation stage (iii). However, unloading commencing from $B$ is commonly associated with evidence of an existing state of prestrain. That pre-strain state becomes evident, and, hence, measurable in the form of residual strain as soon as loading is completely removed at some subsequent material configuration, $C$ (Figure 1).

Mathematical modelling is simplified if $C$, rather than $B$, is temporarily considered as the point of principal reference of the elastic deformation stage (iii). Indeed, in a limiting situation where the points $C$ and $B$ shown in Figure 1 approach simultaneously the points $O$ and $A$, respectively, the elastic deformation stage (iii) degenerates into the initial elastic stage (i) discussed previously in Section 3. Stage (i) is accordingly perceived as a particular case of the more general elastic deformation stage (iii), in which the completely unloaded deformation state, $O$, is free of residual strain.

Most of the definitions and equations associated with the pilot problem introduced in Section 2 are thus seen still valid in stage (iii), as soon as all symbols representing time, position and deformation are generalised by adding upon them a so-called "hat". An adequate notation extension of all equations and relevant relations met previously in the elastic stage (i) can accordingly be summarised as follows:

$$
\begin{aligned}
& \hat{x}_{1}=\hat{X}_{1} \frac{\hat{t}}{\hat{t}_{0}}, \hat{x}_{2}=\hat{X}_{2}, \quad \hat{x}_{3}=\hat{X}_{3}, \\
& \hat{L}=\left(\hat{t} / \hat{t}_{0}\right) \hat{L}_{\hat{0}}, \quad-\hat{L}_{\hat{0}} / 2 \leq \hat{X}_{1} \leq \hat{L}_{\hat{0}} / 2, \\
& -1 / 2 \leq \hat{X}_{2}, \hat{X}_{3} \leq 1 / 2=\hat{L}_{0} / 2, \\
& \hat{\boldsymbol{F}}=\operatorname{diag}\left\{\hat{t} / \hat{t}_{0}, 1,1\right\}, \hat{\boldsymbol{C}}=\operatorname{diag}\left\{\left(\hat{t} / \hat{t}_{0}\right)^{2}, 1,1\right\}, \\
& \hat{\rho}=\hat{\rho}_{\hat{0}} \hat{t}_{0} / \hat{t},
\end{aligned}
$$

where,

$$
\left.\hat{\boldsymbol{F}}\right|_{\hat{t}=\widehat{t}_{0}}=\left.\hat{\boldsymbol{C}}\right|_{\hat{t}=\hat{t}_{0}}=\mathbf{I} \text {, }
$$

and a suffix " $\hat{0}$ " indicates quantities measured/determined at $\hat{t}=\hat{t}_{0} \equiv \hat{t}_{C}$.

In this context, the anticipated residual strain observed at $\hat{t}_{0}$ will be represented by the tensor quantity $\hat{\boldsymbol{C}}_{\hat{0}}\left(\neq\left.\hat{\boldsymbol{C}}\right|_{\hat{t}=\hat{t}_{0}}\right)$. The relevant residual stress tensor that may also be encountered at $\hat{t}_{0}$ will be denoted with $\hat{\boldsymbol{T}}_{\hat{0}}$. These tensor quantities are determined in the manner detailed in Section 6.3 below. Their involvement in the observed deformation is regarded as a manifestation of the fact that, at $\hat{t}=\hat{t}_{0} \neq t_{0}$, the material of interest is not expected to recover its initial cubic shape. 
The currently unknown cuboidal shape emerging at $\hat{t}_{0}$ is accordingly regarded as a new (or updated) initial configuration, described in the new (or updated) Cartesian co-ordinate system $\hat{X}_{R}$. $\hat{\boldsymbol{C}}_{\hat{0}}$ is then regarded as the right Cauchy-Green deformation tensor that relates, in a static deformation manner, the initial unit cube form $\left(t=t_{0}\right)$ with that cuboid deformation state encountered at $\hat{t}=\hat{t}_{0}$. Consideration of the particular case $\hat{t} \equiv t$ leads then to the following initial conditions:

$\left.\hat{\boldsymbol{C}}_{\hat{0}}\right|_{\hat{t} \equiv t}=\mathbf{I},\left.\quad \hat{\boldsymbol{T}}_{\hat{0}}\right|_{\hat{t} \equiv t}=\mathbf{0}$,

$\left.\hat{L}_{\hat{0}}\right|_{\hat{t} \equiv t}=L_{0}=1$.

It is emphasised (see also Section 6.3 below) that $\hat{t}=\hat{t}_{B}$ is regarded as the end of continuous loading from, rather than the beginning of continuous unloading towards $\hat{t}=\hat{t}_{0}$ only for mathematical modelling purposes. In fact, continuous loading from $\hat{t}=\hat{t}_{C} \equiv \hat{t}_{0}$ brings the material back to the deformation state encountered previously at $t=t_{B} \equiv \hat{t}_{B}$. Further loading will then direct the assumed deformation back into its work-hardening track (ii), where the generalised time parameter, $\hat{t}$, is naturally replaced by $t$. In accordance with an earlier relevant argument (see Section 2), $\hat{t}$ is thus also perceived, along with $t$, as a parameter that accounts for succession of events, rather than, strictly, as a parameter that represents real time.

It is worth noting that the quantities appearing in (6.1) have all obtained particular forms that serve the pilot problem introduced in Section 2. However, the general notation and relevant concepts employed in this Section are applicable more generally, regardless of particularly adopted forms of the imposed deformation pattern.

\subsection{Constitutive equations}

At $\hat{t}=\hat{t}_{0}$, when the initial conditions (6.2) hold, $\hat{\boldsymbol{C}}_{\hat{0}}$ and $\hat{\boldsymbol{T}}_{\hat{0}}$ are still related directly through the constitutive equations sought; namely (6.8) below. The strain energy density of the material may be represented in stage (iii) in the following dual form:

$W^{I I I}\left(\hat{\boldsymbol{C}}, \hat{\boldsymbol{C}}_{\hat{0}}, \boldsymbol{e}_{1}\right)=\tilde{W}^{I I I}\left(\hat{\boldsymbol{C}}, \hat{\boldsymbol{T}}_{\hat{0}}, \boldsymbol{e}_{1}\right)$.

This implies that, for mathematical modelling purposes, the material can be considered in stage (iii) as either residually strained or residually stressed. Moreover, (6.4) implies that, unless $\hat{t} \equiv t, \boldsymbol{e}_{1}$ still defines a material direction of permanent transverse isotropy.

Application of the power balance procedure outlined in Appendix A again leads to the standard hyperelasticity constitutive equation. In the light of (6.4), this is expressed in a dual form as follows:

$\sigma_{i j}^{I I I}=\frac{\hat{\rho}}{\hat{\rho}_{\hat{0}}} \hat{F}_{i R} \hat{F}_{j S}\left(\frac{\partial W^{I I I}}{\partial \hat{C}_{R S}}+\frac{\partial W^{I I I}}{\partial \hat{C}_{S R}}\right)=\frac{\hat{\rho}}{\hat{\rho}_{\hat{0}}} \hat{F}_{i R} \hat{F}_{j S}\left(\frac{\partial \widetilde{W}^{I I I}}{\partial \hat{C}_{R S}}+\frac{\partial \widetilde{W}^{I I I}}{\partial \hat{C}_{S R}}\right)$,

where either of $W^{I I I}$ and $\tilde{W}^{I I I}$ is required to be a function of the components of a corresponding complete set of relevant deformation invariants. As $W^{I I I}$, for instance, is a function of the agents appearing in the left hand side of (6.4), such a set is obtained in a straightforward manner by replacing the tensors $\boldsymbol{C}, \boldsymbol{D}^{p}$ and $\boldsymbol{A}$ appearing in (A.8) with $\hat{\boldsymbol{C}}, \hat{\boldsymbol{C}}_{\hat{0}}$ and $\boldsymbol{e}_{1}$, respectively, thus leading to 


$$
\begin{aligned}
& I_{1}^{I I I}=\operatorname{tr} \hat{\boldsymbol{C}}, I_{2}^{I I I}=\frac{1}{2}\left[(\operatorname{tr} \hat{\boldsymbol{C}})^{2}-\operatorname{tr} \hat{\boldsymbol{C}}^{2}\right], I_{3}^{I I I}=\operatorname{det} \hat{\boldsymbol{C}}, I_{4}^{I I I}=\boldsymbol{e}_{1}^{T} \hat{\boldsymbol{C}}_{1}=\hat{\boldsymbol{C}}_{11}, I_{5}^{I I I}=\boldsymbol{e}_{1}^{T} \hat{\boldsymbol{C}}^{2} \boldsymbol{e}_{1}=\hat{\boldsymbol{C}}_{11}^{2}, \\
& I_{6}^{I I I}=\operatorname{tr} \hat{\boldsymbol{C}} \hat{\boldsymbol{C}}_{\hat{0}}, I_{7}^{I I I}=\operatorname{tr} \hat{\boldsymbol{C}} \hat{\boldsymbol{C}}_{\hat{0}}^{2}, I_{8}^{I I I}=\operatorname{tr} \hat{\boldsymbol{C}}^{2} \hat{\boldsymbol{C}}_{\hat{0}}, I_{9}^{I I I}=\operatorname{tr} \hat{\boldsymbol{C}}^{2} \hat{\boldsymbol{C}}_{\hat{0}}^{2}, I_{10}^{I I I}=\boldsymbol{e}_{1}^{T} \hat{\boldsymbol{C}} \hat{\boldsymbol{C}}_{\hat{0}} \boldsymbol{e}_{1}=\hat{C}_{11} \hat{\boldsymbol{C}}_{\hat{0} 11}, \\
& I_{11}^{I I I}=\operatorname{tr} \hat{\boldsymbol{C}}_{\hat{0}}, I_{12}^{I I I}=\operatorname{tr} \hat{\boldsymbol{C}}_{\hat{0}}^{2}, I_{13}^{I I I}=\operatorname{tr} \hat{\boldsymbol{C}}_{\hat{0}}^{3}, I_{14}^{I I I}=\boldsymbol{e}_{1}^{T} \hat{\boldsymbol{C}}_{\hat{0}} \boldsymbol{e}_{1}=\hat{\boldsymbol{C}}_{\hat{0} 11}, I_{15}^{I I I}=\boldsymbol{e}_{1}^{T} \hat{\boldsymbol{C}}_{\hat{0}}^{2} \boldsymbol{e}_{1}=\hat{\boldsymbol{C}}_{\hat{0} 11}^{2} .
\end{aligned}
$$

Further development of (6.5a) becomes then possible by expanding that form of the constitutive equation as follows:

$$
\sigma_{i j}^{I I I}=\frac{\hat{\rho}}{\hat{\rho}_{\hat{0}}} \hat{F}_{i R} \hat{F}_{j S} \sum_{n=1}^{15} \frac{\partial W^{I I I}}{\partial I_{n}^{I I I}}\left(\frac{\partial I_{n}^{I I I}}{\partial \hat{C}_{R S}}+\frac{\partial I_{n}^{I I I}}{\partial \hat{C}_{S R}}\right)=\frac{\hat{\rho}}{\hat{\rho}_{\hat{0}}} \hat{F}_{i R} \hat{F}_{j S} \sum_{n=1}^{10} \frac{\partial W^{I I I}}{\partial I_{n}^{I I I}}\left(\frac{\partial I_{n}^{I I I}}{\partial \hat{C}_{R S}}+\frac{\partial I_{n}^{I I I}}{\partial \hat{C}_{S R}}\right) .
$$

Through a process similar to that outlined in Appendix A, this finally leads to

$$
\begin{aligned}
\sigma_{i j}^{I I I}=2 \frac{\hat{\rho}}{\hat{\rho}_{\hat{0}}} \hat{F}_{i R} \hat{F}_{j S}\left\{\left(W_{1}^{I I I}+I_{1}^{I I I} W_{2}^{I I I}+I_{2}^{I I I} W_{3}^{I I I}\right) \delta_{R S}-\left(W_{2}^{I I I}+I_{1}^{I I I} W_{3}^{I I I}\right) \hat{C}_{R S}+W_{3}^{I I I} \hat{C}_{R P} \hat{C}_{P S}\right\} \\
+2 \frac{\hat{\rho}}{\hat{\rho}_{\hat{0}}}\left\{\hat{F}_{i 1} \hat{F}_{j 1} W_{4}^{I I I}+W_{5}^{I I I}\left(\hat{F}_{i 1} \hat{F}_{j S} \hat{C}_{S 1}+\hat{F}_{i R} \hat{F}_{j 1} \hat{C}_{R 1}\right)+\hat{F}_{i 1} \hat{F}_{j S} W_{10}^{I I I} \hat{C}_{\hat{0} S 1}\right\}+ \\
+2 \frac{\hat{\rho}}{\hat{\rho}_{\hat{0}}} \hat{F}_{i R} \hat{F}_{j S}\left\{W_{6}^{I I I} \hat{C}_{\hat{0} R S}+W_{7}^{I I I} \hat{C}_{\hat{0} R P} \hat{C}_{\hat{0} P S}+2\left(W_{8}^{I I I} \hat{C}_{\hat{0} R P}+W_{9}^{I I I} \hat{C}_{\hat{0} R Q} \hat{C}_{\hat{0} Q P}\right) \hat{C}_{P S}\right\},
\end{aligned}
$$

where

$$
W_{\ell}^{I I I}=\frac{\partial W^{I I I}}{\partial I_{\ell}^{I I I}}(\ell=1,2, \ldots, 10) .
$$

When connected with (6.2) at $\hat{t}=\hat{t}_{0},(6.8)$ leads to

$$
\begin{aligned}
\left.T_{\hat{0} i j} \equiv \sigma_{i j}^{I I I}\right|_{\hat{i}=\hat{t}_{0}}=2\left\{\left(W_{\hat{0} 1}^{I I I}+2 W_{\hat{0} 2}^{I I I}+W_{\hat{0} 3}^{I I I}\right) \delta_{i j}+\left(W_{\hat{0} 4}^{I I I}+2 W_{\hat{0} 5}^{I I I}\right) \delta_{i 1} \delta_{j 1}\right. \\
\left.+\left(W_{\hat{0} 6}^{I I I}+2 W_{\hat{0} 8}^{I I I}\right) \hat{C}_{\hat{0} i j}+\left(W_{\hat{0} 7}^{I I I}+2 W_{\hat{0} 9}^{I I I}\right) \hat{C}_{\hat{0} i k} \hat{C}_{\hat{0} k j}+W_{\hat{0} 10}^{I I I} \delta_{i 1} \hat{C}_{\hat{0} j 1}\right\},
\end{aligned}
$$

which is the previously implied relation that underpins the dual representation (6.4) of the strain energy density. Use of (6.4b) may accordingly lead to an alternative form of the constitutive equation (6.8), in which $\hat{\boldsymbol{C}}_{\hat{0}}$ will essentially be replaced by $\hat{\boldsymbol{T}}_{\hat{0}}$.

\subsection{Compatibility with the elastic deformation stage (i)}

In the particular case that stage (iii) degenerates to its isotropic elastic counterpart (i), $\boldsymbol{e}_{1}$ does not anymore represent a preferred material direction, and, because (6.3a) also holds, it is seen that

$$
\begin{aligned}
& \left.I_{6}^{I I I}\right|_{\hat{t}=t}=\left.I_{7}^{I I I}\right|_{\hat{t}=t}=\operatorname{tr} \hat{\boldsymbol{C}}=I_{1}^{I I I} \equiv I_{1}^{I}, \\
& \left.I_{8}^{I I I}\right|_{\hat{t}=t}=\left.I_{9}^{I I I}\right|_{\hat{t} \equiv t}=\operatorname{tr} \hat{\boldsymbol{C}}^{2}=\left(I_{1}^{I I I}\right)^{2}-2\left(I_{2}^{I I I}\right)^{2} \equiv\left(I_{1}^{I}\right)^{2}-2\left(I_{2}^{I}\right)^{2}, \\
& \left.I_{11}^{I I I}\right|_{\hat{t}=t}=\left.I_{12}^{I I I}\right|_{\hat{t}=t}=\left.I_{13}^{I I I}\right|_{\hat{t}=t}=3,
\end{aligned}
$$

The deformation invariants $\hat{I}_{\ell}(\ell=4,5, \ldots, 15)$ become redundant in stage (i), where the first part of the continuity condition $(2.7 \mathrm{c})$ imposes the requirement

$$
\left.W^{I I I}\right|_{\hat{t} \equiv t}=W^{I} \text {. }
$$

Moreover, connection of (6.11) with the constitutive equation (6.8) leads to 


$$
\begin{aligned}
\left.\sigma_{i j}^{I I I}\right|_{\hat{\imath}=t}=2 \frac{\rho}{\rho_{0}} F_{i R} F_{j S}\left\{\left(W_{1}^{I I I}+I_{1}^{I} W_{2}^{I I I}+I_{2}^{I} W_{3}^{I I I}+W_{6}^{I I I}+W_{7}^{I I I}\right) \delta_{R S}\right. \\
\left.\left.-\left[W_{2}^{I I I}+I_{1}^{I} W_{3}^{I I I}-2\left(W_{8}^{I I I}+W_{9}^{I I I}\right)\right] C_{R S}-W_{3}^{I I I} C_{R P} C_{P S}\right\}\right\}_{\hat{i}=t} \\
+2 \frac{\rho}{\rho_{0}}\left\{F_{i 1} F_{j 1}\left(W_{4}^{I I I}+W_{10}^{I I I}\right)+W_{5}^{I I I}\left(F_{i 1} F_{j S} C_{S 1}+F_{i R} F_{j 1} C_{R 1}\right)\right\}_{\hat{t}=t},
\end{aligned}
$$

which, by virtue of the first part of (2.8c), is required to be identical with its stage (i) counterpart, namely (3.3). Hence, a comparison of (6.13) and (3.3) provides the following compatibility conditions:

$$
\begin{aligned}
& \left.W_{3}^{\text {III }}\right|_{\hat{t}=t}=W_{3}^{I}, \\
& {\left[W_{2}^{I I I}-2\left(W_{8}^{I I I}+W_{9}^{I I I}\right)\right]_{\hat{t} \equiv t}=W_{2}^{I},} \\
& {\left[W_{1}^{I I I}+I_{1} W_{2}^{I I I}+W_{6}^{I I I}+W_{7}^{I I I}\right]_{\hat{t}=t}=W_{1}^{I}+I_{1} W_{2}^{I},} \\
& W_{4}^{I I I}+W_{10}^{I I I}=0, \\
& W_{5}^{I I I}=0 .
\end{aligned}
$$

The last two of (6.14) reveal that $W^{I I I}$ should be independent of $I_{5}^{I I I}$, and such that $W_{10}^{I I I}=-W_{4}^{I I I}$. The constitutive equations (6.8) and (6.10) can thus slightly simplify, and become

$$
\begin{gathered}
\sigma_{i j}^{I I I}=2 \frac{\hat{\rho}}{\hat{\rho}_{\hat{0}}}\left\{\hat { F } _ { i R } \hat { F } _ { j S } \left[\left(W_{1}^{I I I}+I_{1}^{I I I} W_{2}^{I I I}+I_{2}^{I I I} W_{3}^{I I I}\right) \delta_{R S}-\left(W_{2}^{I I I}+I_{1}^{I I I} W_{3}^{I I I}\right) \hat{C}_{R S}+W_{3}^{I I I} \hat{C}_{R P} \hat{C}_{P S}+\right.\right. \\
\left.W_{4}^{I I I} \hat{F}_{i 1}\left(\hat{F}_{j 1}-\hat{F}_{j S} \hat{C}_{\hat{0} S 1}\right)+\hat{F}_{i R} \hat{F}_{j S}\left[W_{6}^{I I I} \hat{C}_{\hat{0} R S}+W_{7}^{I I I} \hat{C}_{\hat{0} R P} \hat{C}_{\hat{0} P S}+2\left(W_{8}^{I I I} \hat{C}_{\hat{0} R P}+W_{9}^{I I I} \hat{C}_{\hat{0} R Q} \hat{C}_{\hat{0} Q P}\right) \hat{C}_{P S}\right]\right\}, \\
\left.T_{\hat{0} i j} \equiv \sigma_{i j}^{I I I}\right|_{\hat{i}=\hat{t}_{0}}=2\left\{\left(W_{\hat{0} 1}^{I I I}+2 W_{\hat{0} 2}^{I I I}+W_{\hat{0} 3}^{I I I}\right) \delta_{i j}+W_{\hat{0} 4}^{I I I} \delta_{i 1}\left(\delta_{j 1}-\hat{C}_{\hat{0} j 1}\right)+\right. \\
\left.\left(W_{\hat{0} 6}^{I I I}+2 W_{\hat{0} 8}^{I I I}\right) \hat{C}_{\hat{0} i j}+\left(W_{\hat{0} 7}^{I I I}+2 W_{\hat{0} 9}^{I I I}\right) \hat{C}_{\hat{0} i k} \hat{C}_{\hat{0} k j}+W_{\hat{0} 10}^{I I I} \delta_{i 1} \hat{C}_{\hat{0} j 1}\right\},
\end{gathered}
$$

where the compatibility conditions (6.12) and (6.14a-c) are still required to hold. These suggest that there still exists considerable mutual dependency between the forms of $W^{I I I}$ and $W^{I}$.

\subsection{Compatibility with the work-hardening stage (ii) - Determination of residual strain}

Because continuous reloading from $\hat{t}=\hat{t}_{0}$ brings the material back into the state encountered previously at $\hat{t}=\hat{t}_{B}$ where (6.3) holds, the stress continuity condition (2.8b) obtains the following alternative form,

$\left.\sigma_{i j}^{I I I}\right|_{\hat{t}=\hat{t}_{B} \equiv t_{B}}=\left.\sigma_{i j}^{I I}\right|_{t=t_{B} \equiv \hat{t}_{B}}$.

The right hand side of this condition is considered known from the analysis detailed previously in Section 5. Connection of (6.16) with (6.8) leads then to

$$
\begin{aligned}
& {\left[\frac{\hat{\rho}}{\hat{\rho}_{\hat{0}}} \hat{F}_{i R} \hat{F}_{j S}\left\{W_{6}^{I I I} \hat{C}_{\hat{0} R S}+W_{7}^{I I I} \hat{C}_{\hat{0} R P} \hat{C}_{\hat{0} P S}+2\left(W_{8}^{I I I} \hat{C}_{\hat{0} R P}+W_{9}^{I I I} \hat{C}_{\hat{0} R Q} \hat{C}_{\hat{0} Q P}\right) \hat{C}_{P S}\right\}-\frac{\hat{\rho}}{\hat{\rho}_{\hat{0}}} \hat{F}_{i 1} \hat{F}_{j S} W_{4}^{I I I} \hat{C}_{\hat{0} S 1}+\right.} \\
& \left.\frac{\hat{\rho}}{\hat{\rho}_{\hat{0}}} \hat{F}_{i R} \hat{F}_{j S}\left\{\left(W_{1}^{I I I}+I_{1}^{I I I} W_{2}^{I I I}+I_{2}^{I I I} W_{3}^{I I I}\right) \delta_{R S}-\left(W_{2}^{I I I}+I_{1}^{I I I} W_{3}^{I I I}\right) \hat{C}_{R S}+W_{3}^{I I I} \hat{C}_{R P} \hat{C}_{P S}\right\}+\frac{\hat{\rho}}{\hat{\rho}_{\hat{0}}} \hat{F}_{i 1} \hat{F}_{j 1} W_{4}^{I I I}\right]_{\hat{\hat{l}}=\hat{t}_{B}=t_{B}} \\
& =\left.\sigma_{i j}^{I I I}\right|_{t=t_{B}=\hat{t}_{B}},
\end{aligned}
$$

which represent six simultaneous algebraic equations for the same number of unknown components of the residual strain tensor $\hat{\boldsymbol{C}}_{\hat{0}}$.

For dilatational type deformations, the diagonal form of all tensor quantities involved in the analysis reveals that three of these equations refer to non-diagonal components of $\hat{\boldsymbol{C}}_{\hat{0}}$, and are thus satisfied identically. The remaining three equations are obtained by allowing both free indices appearing in (6.17) to 
acquire simultaneously the values 1,2 or 3 . These are generally adequate for the determination of all three diagonal components of $\hat{\boldsymbol{C}}_{\hat{0}}$. Nevertheless, due to the symmetries of the deformation considered in this investigation, it is anticipated that $\hat{C}_{\hat{0} 22}=\hat{C}_{\hat{0} 33}$ and, hence, that the last two of the implied algebraic equations are equivalent.

Moreover, in the particular case of the present pilot problem, the transverse edges of the deforming cuboid remain unstrained. Hence, a comparison of the deformation states attained at $t_{0}$ and $\hat{t}_{0}$ reveals that $\hat{C}_{\hat{0} 11}=\left(\hat{L}_{\hat{0}} / L_{0}\right)^{2}, \quad \hat{C}_{\hat{0} 22}=\hat{C}_{\hat{0} 33}=1$.

Because $\hat{C}_{\hat{0} 22}=\hat{C}_{\hat{0} 33}$ is thus prescribed in this case, the aforementioned couple of equivalent algebraic equations are either satisfied identically or unable to provide some additional information regarding the cuboid material response. It follows that the last remaining algebraic equation, obtained by setting both free indices appearing in (6.17) equal to 1 , suffices for the determination of $\hat{C}_{\hat{0} 11}$ or, equivalently, $\hat{L}_{\hat{0}}$.

Validity of $(6.1 \mathrm{~b}, \mathrm{c})$ and/or $(6.18 \mathrm{~b}, \mathrm{c})$ makes it clear that, in this particular case, the conditions of deformation continuity mentioned after (2.8) degenerate into the single equation

$\left.L\right|_{t=t_{B} \equiv \hat{t}_{B}}=\left.\hat{L}\right|_{\hat{t}=\hat{t}_{B} \equiv t_{B}}$.

By virtue of (3.7) and (6.1d), it is also

$\hat{L}_{\hat{0}} / L_{0}=\hat{t}_{0} / t_{0}$,

and, because $t_{0}$ is considered known, determination of $\hat{L}_{\hat{0}}$ also provides the value of the parameter $\hat{t}_{0}$ at which the deforming cuboid is unloaded in stage (iii).

The externally applied forces that maintain the implied deformation within the elastic deformation regime (iii) can thus be determined as follows

$\hat{f}=\sigma_{11}^{\text {III }} L_{0}^{2}$,

$f=\sigma_{22}^{I I I}\left(\hat{t} / \hat{t}_{0}\right) \hat{L}_{\hat{0}} L_{0}=\sigma_{33}^{I I I}\left(\hat{t} / \hat{t}_{0}\right) \hat{L}_{\hat{0}} L_{0}$.

\subsection{Application: Residually strained deformation of a compressible Mooney-Rivlin cube}

One of the simplest possible extensions that the the Mooney-Rivlin strain energy density considered earlier in Section 3.2 can attain during the deformation stage (iii) is as follows:

$$
W^{I I I}(\boldsymbol{C})=\frac{\mu_{1}}{2}\left(I_{1}^{I I I}-3\right)+\frac{\mu_{2}}{2}\left(I_{2}^{I I I}-3\right)+\frac{\mu_{B}}{2}\left(I_{4}^{I I I}-1\right)-\frac{\mu_{B}}{2}\left(I_{10}^{I I I}-\left.I_{10}^{I I I}\right|_{\hat{t}=\hat{t}_{0}}\right) \text {. }
$$

This form of $W^{I I I}$ does satisfy the compatibility conditions (6.12) and (6.14) and involves a new material parameter, $\mu_{B}$. As is implied by the associated suffix, the value of this parameter may depend on deformation characteristics related to the offset yield point $B$, including the value of the parameter $t_{B}$. When connected with the deformation pattern (6.1) of the present pilot dilatation problem, (6.22) reduces to

$$
W^{I I I}(\boldsymbol{C})=\left(\frac{\mu_{1}}{2}+\mu_{2}\right)\left(\hat{C}_{11}-1\right)-\frac{\mu_{B}}{2}\left(\hat{C}_{11}-1\right)\left(\hat{C}_{\hat{0} 11}-1\right) \text {, }
$$

which evidently holds in connection with (6.18).

Connection of (6.22) with the algebraic equations (6.17) enables the latter to simplify as follows:

$$
\left[\frac{\hat{\rho}}{\hat{\rho}_{\hat{0}}} \hat{F}_{i R} \hat{F}_{j S}\left(\left(\mu_{1}+I_{1}^{I I I} \mu_{2}\right) \delta_{R S}-\mu_{2} \hat{C}_{R S}\right\}+\frac{\hat{\rho}}{\hat{\rho}_{\hat{0}}} \mu_{B} \hat{F}_{i 1}\left(\hat{F}_{j 1}-\hat{F}_{j S} \hat{C}_{\hat{0} S 1}\right)\right]_{\hat{t}=\hat{t}_{B} \equiv t_{B}}=\left.\sigma_{i j}^{I I}\right|_{t=t_{B}} \text {. }
$$

If the appearing free indices, $i$ and $j$, are set simultaneously equal to either 2 or 3 , then (6.24) returns 


$$
\left(\mu_{1}+\mu_{2}\right)+\left(\hat{t}_{B} / \hat{t}_{0}\right)^{2} \mu_{1}=-\lambda^{-1} \beta_{3}-\left.\left(\hat{t}_{B} / \hat{t}_{0}\right)\left[\lambda^{-1} \beta_{1}\left(t_{B} / t_{0}\right)-\beta_{2}\right] \sigma_{11}^{I I}\right|_{t=t_{B}},
$$

where (5.18) is also accounted for, and

$$
\left.\sigma_{11}^{I I}\right|_{t=t_{B}}=\frac{1}{K\left(t_{B}\right)}\left\{\left(t_{A} / t_{0}\right)\left(\mu_{1}+2 \mu_{2}\right) K\left(t_{A}\right)+\int_{t_{A}}^{t_{B}} K(t) q(t) d t\right\},
$$

by virtue of (5.23) and (5.22).

Consideration of (5.19) and (5.20) then reveals that, unless it is satisfied identically, (6.25) emerges as a second equation, along with (4.20), that relates all the material parameters appearing in the particular forms of $W^{I}, W^{I I}$ and $\varphi^{I I}$ employed in this pilot application. It follows that, after (5.19) and (5.20) are inserted into (6.25), the resulting equation and (4.20) form a pair of simultaneous algebraic equations for a particular pair of the involved parameters; such as the pair $\alpha_{1}$ and $\alpha_{2}$ involved in the yield function. This observation makes it more generally understood that the form that the yield function attains within the workhardening stage (ii) is indirectly connected with its $W^{I}$ and $W^{I I}$ counterparts.

Moreover, by setting the value of both free indices appearing in (6.24) equal to 1 , one obtains

$\left(t_{B} / \hat{t}_{0}\right)\left[\mu_{1}+2 \mu_{2}+\mu_{B}\left(1-\hat{C}_{\hat{0} 11}\right)\right]=\left.\sigma_{11}^{I I}\right|_{t=t_{B}}$,

which, with use of (6.18a) reduces to the quadratic equation

$\hat{\mu}\left(\hat{L}_{\hat{0}} / L_{0}\right)^{2}+\left(\left.\sigma_{11}^{I I}\right|_{t=t_{B}}\right)\left(t_{0} / t_{B}\right)\left(\hat{L}_{\hat{0}} / L_{0}\right)-\left(\mu_{1}+2 \mu_{2}+\mu_{B}\right)=0$.

Solution of this equation will provide the unknown length of the longitudinal edges of the cuboid after unloading is completed in stage (iii). Appendix B outlines some interesting observations that relate the roots of (6.28) with the material moduli involved in this application.

With $\hat{L}_{\hat{0}}$ and, hence, $\hat{t}_{0}$ being thus determined, a combination of (6.15a) with (6.1) and (6.22) provides the stress state in stage (iii) as follows:

$$
\begin{aligned}
& \left.\left.\sigma_{11}^{I I I}=\left(\hat{t} / \hat{t}_{0}\right)\left(\mu_{1}+2 \mu_{2}\right)+\mu_{B} \mid 1-\left(\hat{L}_{\hat{0}} / L_{0}\right)^{2}\right]\right\} \\
& \sigma_{22}^{I I I}=\sigma_{33}^{I I I}=\left(\hat{t}_{0} / \hat{t}\right)\left\{\mu_{1}+\left[\left(\hat{t} / \hat{t}_{0}\right)^{2}+1\right] \mu_{2}\right\}
\end{aligned}
$$

This depends only on time and, hence, still satisfies identically the quasi-static equations of motion (2.6). The external forces that maintain the assumed deformation in stage (iii) are thus found to be

$$
\begin{aligned}
& \left.\hat{f}=L_{0}^{2}\left(\hat{t} / \hat{t}_{0}\right)\left\{\left(\mu_{1}+2 \mu_{2}\right)+\mu_{B} \mid 1-\left(\hat{L}_{\hat{0}} / L_{0}\right)^{2}\right]\right\} \\
& f=\left\{\mu_{1}+\left[\left(\hat{t} / \hat{t}_{0}\right)^{2}+1\right] \mu_{2}\right\} L_{0} \hat{L}_{\hat{0}} .
\end{aligned}
$$

A final observation refers to the fact that, at $\hat{t}=\hat{t}_{0},(6.29)$ returns

$$
\begin{aligned}
& \left.T_{\hat{0} 11} \equiv \sigma_{11}^{I I I}\right|_{\hat{t}=\hat{t}_{0}}=\left\{\left(\mu_{1}+2 \mu_{2}\right)+\mu_{B}\left[1-\left(\hat{L}_{\hat{0}} / L_{0}\right)^{2}\right]\right\}, \\
& T_{\hat{0} 22} \equiv T_{\hat{0} 33}=\left.\sigma_{22}^{I I I}\right|_{\hat{t}=\hat{t}_{0}}=\left(\mu_{1}+2 \mu_{2}\right),
\end{aligned}
$$

which suggest that the externally unloaded cuboid seems to be controversially at a state of residual stress. It is, however, also observed that in the particular case that $\hat{t}_{0}=t_{0}, \hat{L}_{\hat{0}} / L_{0}=1$, and, hence, stage (iii) coincides with stage (i), the stresses (6.29) reduce naturally into their stage (i) counterparts (3.15).

It is thus concluded that, in analogy with the observations detailed in Section 3.2, the completely unloaded, but residually strained elastic cuboid can begin to experience again the non-uniform dilatation (6.1) only after re-loading in stage (iii) allows the material to reach the pre-stress level (6.31). The loading parameter appearing in Figure 1 should accordingly be redefined as follows:

$$
P=\max \left\{\left|\sigma_{11}^{I I I}-T_{\hat{0} 11}\right|,\left|\sigma_{22}^{I I I}-T_{\hat{0} 22}\right|\right\} \text {, }
$$

thus leading to the following generalisation of (3.16): 


$$
P=\max \left\{\left(\hat{t} / \hat{t}_{0}-1\right)\left|\mu_{1}+2 \mu_{2}+\mu_{B}\left[1-\left(\hat{L}_{\hat{0}} / L_{0}\right)^{2}\right],\right|\left(\hat{t}_{0} / \hat{t}-1\right) \mu_{1}+\left[\left(\hat{t} / \hat{t}_{0}\right)+\left(\hat{t}_{0} / \hat{t}\right)-2\right] \mu_{2} \mid\right\}
$$

\section{Conclusions}

The analytical solution of the particular application introduced in Section 3.2, and continued afterwards in Sections 4.4, 5.2 and 6.4, demonstrated comprehensively most of the principal features of the presented plasticity model, which emerges as a powerful new mathematical tool in theoretical solid mechanics. Dealing with a deformable isotropic cube whose initial elastic behaviour is characterised by the strain energy density of a compressible Rivlin-Mooney material, that pilot application met no difficulty to identify compatible strain energy density branches that make accountable either the plastic flow emerging during loading beyond initial yield, or the residual strain encountered after unloading from an offset yield point is completed. Cases in which the initial hyperelastic cube is made by some different isotropic material can be handled in a similar manner, as soon as a corresponding strain energy density is identified.

The outlined analytical solution is in agreement with, and, hence, supports the observation that the initial, pre-strain free, elastic deformation stage (i) is a particular case of any of the subsequent pre-strained elastic deformation stage (iii). Moreover, this solution deals successfully with the physical observation that induced anisotropy caused by large elastic deformation in stage (i) is necessarily converted into irreversible permanent anisotropy when loading continues beyond the initial yield point within the work-hardening stage (ii).

It is thus verified that the proposed plasticity model does not contradict or dismiss any of the known, fundament concepts and features observed in isotropic plasticity. The model is accordingly still based on standard yield function concepts and yield condition postulates. The latter are however properly modified in a manner that enables permanent anisotropy observed beyond initial yield to be accounted for. This is achieved by appropriately refining the complete set of stress invariants that enter the form of the yield function employed in the initial deformation stage (i) and, hence, by enabling stage (ii) to use a compatible, properly modified branch of that yield function. Plastic flow and the corresponding flow rule encountered in the workhardening stage (ii) are thus also affected by the proposed refinement of the initial set of the stress invariants.

In a similar manner, the model considers that the elastic parts of material behaviour are governed by a strain energy density that consists three mutually compatible branches. The basic branch underpins standard hyperelastic material behaviour observed before initial yield is reached. The complete and irreducible set of deformation invariants entering the strain energy density in that stage (i) is, however, properly modified beyond initial yield. Along with the encountered deformation induced permanent anisotropy, this modification takes thus also into consideration the plastic flow and the state of pre-strain observed in the deformation stages (ii) and (iii), respectively.

At this early stage of development, the presented plasticity model refers only to behaviour and response of solid materials which are initially isotropic. For the purpose of relative simplicity, discussion was also restricted to basic dilatational deformations that generate initially induced, and afterwards permanently imposed anisotropy of the type observed in transversely isotropic materials. Nevertheless, the hyperelasticity based background of the model allows it to extend and, hence, to embrace plastic behaviour modelling of materials that exhibit properties of more advanced initial anisotropy. The main alterations required in such cases are essentially those referring to the manner in which initial (induced) and final (permanent) anisotropy influences different branches of the involved yield function and strain energy density. Formation and proper implementation of relevant complete sets of deformation and stress state invariants should then, inevitably, be expected to exert additive influence on the resulting analytical and, possibly, computational/numerical relevant efforts.

The complete analytical solution achieved for the pilot application introduced in Section 3.2 made also clear that the presented plasticity model does not require a-priori specification of a work-hardening parameter that characterises plastic progression. Instead, a parameter that describes the manner in which the 
yield function expands during the work-hardening stage emerges naturally during the solution of the particular problem of interest. Hence, the fact that, in the present pilot example, that work-hardening parameter is essentially the magnitude of the externally applied longitudinal force does not come as a surprise. This is, instead, just a natural consequence of the material and geometrical symmetries that characterise this particular cube dilatation problem.

Most importantly, the part of the analytical solution detailed in Section 5.2 verifies another innovative feature of the proposed model. Namely, its ability to determine the required stress and deformation characteristics without a-priori postulation of the manner in which elastic and plastic parts of strain are assembled and produce the total elastic-plastic type of deformation observed. Instead, the amount of strain associated with plastic flow that emerges during the work-hardening stage is determined, in $a$ posteriori manner, through direct time integration of formulas that represent corresponding components of the rate-of-plastic-deformation tensor. In this manner, an estimate is also provided of the time instant that continuing loading within the work-hardening deformation stage (ii) may end through fracture.

Acknowledgement: The author is grateful to one of the anonymous reviewers for exceptionally helpful input.

\section{Appendix A: Constitution during work-hardening of a transversely isotropic material}

The elastic part of deformation that takes place during work-hardening of a deformable solid is initially considered indistinguishable from its plastic counterpart. Regardless of the kind or the degree of material anisotropy involved in the continuum, this is however still considered governed by the usual rate-of-energy equation

$$
\frac{d}{d t} \int_{V} W^{I I} d V=\int_{S} t \cdot v d S
$$

where $V$ is an arbitrary volume of the material in the deformed configuration, surrounded by a closed surface $S$ having outward unit normal $\boldsymbol{n}$. The components of the appearing traction vector, $\boldsymbol{t}$, are given by the Cauchy's formula

$t_{i}=\sigma_{j i}^{I I} n_{j}$.

Applying Reynold's transport theorem and the divergence theorem, one thus obtains

$$
\frac{\rho}{\rho_{0}} \dot{W}^{I I}=\sigma_{j i}^{I I} v_{i, j}+v_{i} \sigma_{j i, j}^{I I}=\sigma_{j i}^{I I} d_{i j} \text {, }
$$

where use is made of the quasi-static equilibrium equations (2.6) and the symmetry of the stress tensor.

The fact that the elastic and the plastic parts of deformation are a-priori indistinguishable is reflected in the postulation

$$
W^{I I}=W^{I I}\left(\boldsymbol{C}, \boldsymbol{D}^{p}\right) \text {, }
$$

where the appearing "pull-back" form of the plastic-rate-of-deformation tensor, $\boldsymbol{D}^{p}$, is defined in the first part of (4.12). This postulation enables the model to account for the dissipative influence that plastic flow exerts on the deformation. It also points out the fact that, by virtue of (4.12), $\boldsymbol{D}^{p}$ is itself a function of the stress tensor and, hence, an indirect function of $\boldsymbol{C}$.

The form (A.4) of $W^{I I}$ implies further that 


$$
\begin{aligned}
\frac{d W^{I I}}{d t} & =\frac{\partial W^{I I}}{\partial C_{R S}} \dot{C}_{R S}+\frac{\partial W^{I I}}{\partial D_{R S}} \dot{D}_{R S}=\left(\frac{\partial W^{I I}}{\partial C_{R S}}+\frac{\partial D_{P N}^{p}}{\partial C_{R S}} \frac{\partial W^{I I}}{\partial D_{P N}^{p}}\right) \dot{C}_{R S} \\
& =\left(\frac{\partial W^{I I}}{\partial C_{R S}}+\frac{\partial D_{P N}^{p}}{\partial C_{R S}} \frac{\partial W^{I I}}{\partial D_{P N}^{p}}\right)\left(\dot{F}_{i R} F_{j S}+F_{i R} \dot{F}_{j S}\right) \\
& =\left(\frac{\partial W^{I I}}{\partial C_{R S}}+\frac{\partial D_{P N}^{p}}{\partial C_{R S}} \frac{\partial W^{I I}}{\partial D_{P N}^{p}}\right)\left(\frac{\partial v_{i}}{\partial X_{R}} \frac{\partial x_{i}}{\partial X_{S}}+\frac{\partial x_{i}}{\partial X_{R}} \frac{\partial v_{i}}{\partial X_{S}}\right) \\
& =\left[\frac{\partial W^{I I}}{\partial C_{R S}}+\frac{\partial W^{I I}}{\partial C_{S R}}+\left(\frac{\partial D_{P N}^{p}}{\partial C_{R S}}+\frac{\partial D_{P N}^{p}}{\partial C_{S R}}\right) \frac{\partial W^{I I}}{\partial D_{P N}^{p}}\right] \frac{\partial x_{i}}{\partial X_{R}} \frac{\partial x_{j}}{\partial X_{S}} \frac{\partial v_{i}}{\partial x_{j}} \\
& =F_{i R} F_{j S}\left[\frac{\partial W^{I I}}{\partial C_{R S}}+\frac{\partial W^{I I}}{\partial C_{S R}}+\left(\frac{\partial D_{P N}^{p}}{\partial C_{R S}}+\frac{\partial D_{P N}^{p}}{\partial C_{S R}}\right) \frac{\partial W^{I I}}{\partial D_{P N}^{p}}\right] d_{i j} .
\end{aligned}
$$

A comparison of (A.3) and (A.5) yields the work-hardening constitutive equation as follows:

$$
\sigma_{i j}^{I I}=\frac{\rho}{\rho_{0}} F_{i R} F_{j S}\left(\frac{\partial W^{I I}}{\partial C_{R S}}+\frac{\partial W^{I I}}{\partial C_{S R}}+2 \frac{\partial D_{P N}^{p}}{\partial C_{R S}} \frac{\partial W^{I I}}{\partial D_{P N}^{p}}\right) \text {. }
$$

This can be developed further in the usual manner, by requiring from $W^{I I}$ to be a function of a complete basis of appropriate deformation invariants. The components of that basis should however also depend on the degree of possible anisotropy that may be observed in the material. If material anisotropy does exist and is due to one or more preference material directions, then (A.6) still holds but the unit vectors that define those directions should also be included among the arguments of $W^{I I}($ e.g., $[16,17])$.

The present case of interest is adequately modelled by considering the following extension of (A.4):

$$
W^{I I}=W^{I I}\left(\boldsymbol{C}, \boldsymbol{D}^{p}, \boldsymbol{A}\right) \text {, }
$$

where the unit vector $\boldsymbol{A}$ defines the preference direction within a transversely isotropic material. The complete and irreducible basis of deformation invariants sought is thus as follows (e.g., [17]):

$$
\begin{aligned}
& I_{1}^{I I}=\operatorname{tr} \boldsymbol{C}, I_{2}^{I I}=\frac{1}{2}\left[(\operatorname{tr} \boldsymbol{C})^{2}-\operatorname{tr} \boldsymbol{C}^{2}\right], I_{3}^{I I}=\operatorname{det} \boldsymbol{C}, I_{4}^{I I}=\boldsymbol{A}^{\boldsymbol{T}} \boldsymbol{C A}, I_{5}^{I I}=\boldsymbol{A}^{\boldsymbol{T}} \boldsymbol{C}^{2} \boldsymbol{A}, \\
& I_{6}^{I I}=\operatorname{tr} \boldsymbol{C} \boldsymbol{D}^{p}, I_{7}^{I I}=\operatorname{tr} \boldsymbol{C}\left(\boldsymbol{D}^{p}\right)^{2}, I_{8}^{I I}=\operatorname{tr} \boldsymbol{C}^{2} \boldsymbol{D}^{p}, I_{9}^{I I}=\operatorname{tr} \boldsymbol{C}^{2}\left(\boldsymbol{D}^{p^{2}}\right), I_{10}^{I I}=\boldsymbol{A}^{T} \boldsymbol{C} \boldsymbol{D}^{p} \boldsymbol{A}, \\
& I_{11}^{I I}=\operatorname{tr} \boldsymbol{D}^{p}, I_{12}^{I I}=\operatorname{tr}\left(\boldsymbol{D}^{p^{2}}\right), I_{13}^{I I}=\operatorname{tr}\left(\boldsymbol{D}^{p^{3}}\right), I_{14}^{I I}=\boldsymbol{A}^{T} \boldsymbol{D}^{p} \boldsymbol{A}, I_{15}^{I I}=\boldsymbol{A}^{T}\left(\boldsymbol{D}^{p^{2}}\right) \boldsymbol{A},
\end{aligned}
$$

and its connection with (A.6) leads to

$$
\sigma_{i j}^{I I}=\frac{\rho}{\rho_{0}} F_{i R} F_{j S}\left\{\sum_{n=1}^{10} \frac{\partial W^{I I}}{\partial I_{n}^{I I}}\left(\frac{\partial I_{n}^{I I}}{\partial C_{R S}}+\frac{\partial I_{n}^{I I}}{\partial C_{S R}}\right)+2 \frac{\partial D_{M N}^{p}}{\partial C_{R S}} \sum_{n=10}^{15} \frac{\partial W^{I I}}{\partial I_{n}} \frac{\partial I_{n}^{I I}}{\partial D_{M N}^{p}}\right\} .
$$

With use of intermediate results of the type

$$
\frac{\partial C_{N N}}{\partial C_{R S}}=\delta_{R S}, \frac{\partial\left(A_{M} C_{M N} A_{N}\right)}{\partial C_{R S}}=A_{R} A_{S}, \frac{\partial\left(C_{N P} C_{P N}\right)}{\partial C_{R S}}=2 C_{R S}, \frac{\partial\left(C_{N P} D_{P N}^{p}\right)}{\partial D_{R S}^{p}}=C_{R S} \text {, etc., }
$$

and, where necessary, of the Cayley-Hamilton theorem, (A.9) is found equivalent to

$$
\begin{aligned}
\sigma_{i j}^{I I}=2 \frac{\rho}{\rho_{0}} F_{i R} F_{j S}\left\{\left(W_{1}^{I I}+I_{1}^{I I} W_{2}^{I I}+I_{2}^{I I} W_{3}^{I I}\right) \delta_{R S}-\left(W_{2}^{I I}+I_{1}^{I I} W_{3}^{I I}\right) C_{R S}+W_{3}^{I I} C_{R P} C_{P S}\right. \\
\left.+W_{4}^{I I} A_{R} A_{S}+W_{5}^{I I}\left(A_{R} C_{S N}+A_{S} C_{R N}\right) A_{N}\right\}+\Theta_{i j}^{I I}(\boldsymbol{\sigma})+\Phi_{i j}^{I I}(\boldsymbol{\sigma}),
\end{aligned}
$$

where 


$$
\begin{aligned}
\Theta_{i j}^{I I}= & 2 \frac{\rho}{\rho_{0}} F_{i R} F_{j S}\left\{W_{6}^{I I} D_{R S}^{p}+W_{7}^{I I} D_{R P}^{p} D_{P S}^{p}+2\left(W_{8}^{I I} D_{R P}^{p}+W_{9}^{I I} D_{R Q}^{p} D_{Q P}^{p}\right) C_{P S}+W_{10}^{I I} A_{R} D_{S N}^{p} A_{N}\right\}, \\
\Phi_{i j}^{I I}= & 2 \frac{\rho}{\rho_{0}} F_{i R} F_{j S} \frac{\partial D_{M N}^{p}}{\partial C_{R S}}\left\{W_{6}^{I I} C_{M N}+2 W_{7}^{I I} C_{M P} D_{P N}^{p}+W_{8}^{I I} C_{M P} C_{P N}+2 W_{9}^{I I} C_{M P} C_{P Q} D_{Q N}^{p}\right. \\
& \left.+W_{10}^{I I} A_{M} C_{N P} A_{P}+W_{11}^{I I} \delta_{M N}+2 W_{12}^{I I} D_{M N}^{p}+3 W_{13}^{I I} D_{M P}^{p} D_{P N}^{p}+W_{14}^{I I} A_{M} A_{N}+2 W_{15}^{I I} A_{M} D_{N P}^{p} A_{P}\right\},
\end{aligned}
$$

and

$$
W_{\ell}^{I I}=\frac{\partial W^{I I}}{\partial I_{\ell}}(\ell=1,2, \ldots, 15) \text {. }
$$

The content of the curly parenthesis in the right-hand-side of (A.11) is identical to the right-hand-side of the constitutive equation met in conventional isotropic hyperelasticity. The new terms, denoted with $\Theta_{i j}^{I I}(\boldsymbol{\sigma})$ and $\Phi_{i j}^{I I}(\boldsymbol{\sigma})$, are both influenced by the plastic rate-of-deformation tensor $\boldsymbol{D}^{p}$ and, by virtue of (4.12), are regarded as stress-feedback terms. It becomes thus understood that, (A.11) is implicit rather than explicit constitutive equation.

In particular, $\Theta_{i j}^{I I}(\boldsymbol{\sigma})$ introduces into the constitutive equation stress-feedback terms that emerge through the direct involvement of $\boldsymbol{D}^{p}$ into the strain energy density. However, the advanced stress-feedback terms that $\Phi_{i j}^{I I}(\boldsymbol{\sigma})$ introduces into the constitutive equation depend not only on $\boldsymbol{D}^{p}$, but are also on $\partial D_{M N}^{p} / \partial C_{R S}$. It becomes thus understood that apart from implicit in the stresses, (A.11) is, in general, a differential, rather than an algebraic constitutive equation.

It is pointed out in this regard that, unless direct evaluation of the terms $\partial D_{M N} / \partial C_{R S}$ can become straightforward in applications, these differential terms may be determined through use of some intermediate variable, $\tilde{q}$ say, which $\boldsymbol{D}$ and $\boldsymbol{C}$ may both depend on; so that

$\partial D_{M N}^{p} / \partial C_{R S}=\frac{\partial D_{M N}^{p}}{\partial \tilde{q}} \frac{\partial \tilde{q}}{\partial C_{R S}}$.

If, as happens for instance in the present case of interest, $\tilde{q}$ is replaced by time, $t$, then

$$
\partial D_{M N}^{p} / \partial C_{R S}=\frac{d D_{M N}^{p}}{d t} \frac{d t}{d C_{R S}}=\dot{D}_{M N}^{p} / \dot{C}_{R S},
$$

which implies that $\Phi_{i j}^{I I}(\boldsymbol{\sigma})$ dependent on the stress components as well as on their time derivatives. In that case, (A.12b) can be expressed in the following form

$$
\begin{aligned}
\Phi_{i j}^{I I}= & 2 \frac{\rho}{\rho_{0}} \frac{F_{i R} F_{j S}}{\dot{C}_{R S}} \dot{D}_{M N}^{p} E_{N M}, \\
E_{N M}= & \left\{W_{6}^{I I} C_{N M}+2 W_{7}^{I I} C_{M P} D_{P N}^{p}+W_{8}^{I I} C_{M P} C_{P N}+2 W_{9}^{I I} C_{M P} C_{P Q} D_{Q N}^{p}\right. \\
& \left.+W_{10}^{I I} A_{M} C_{N P} A_{P}+W_{11}^{I I} \delta_{M N}+2 W_{12}^{I I} D_{N M}^{p}+3 W_{13}^{I I} D_{M P}^{p} D_{P N}^{p}+W_{14}^{I I} A_{M} A_{N}+2 W_{15}^{I I} A_{M} D_{N P}^{p} A_{P}\right\},
\end{aligned}
$$

which is found particularly useful in Section 5.1.

\section{Appendix B: On the roots of the quadratic equation (6.28)}

By requiring from the offset yield point to coincide with the initial yield point and, hence, by setting $t_{B} \equiv t_{A}$ in (6.28), one observes that 
$\hat{L}_{\hat{0}} / L_{0}=1$

becomes a solution of that quadratic algebraic equation. The unit cube representation (B.1) is thus, correctly, a root of (6.28) in that particular case. This result is in agreement with the last of the initial conditions (6.3) and, hence, verifies validity of the modelling claim that deformation stage (i) is a particular case of the residually strained deformation stage (iii).

More generally, solution of the quadratic equation (6.28) returns two possible length values for the longitudinal edges of a completely unloaded cuboid, namely

$\hat{L}_{\hat{0}} / L_{0}=\hat{t}_{0} / t_{0}=\left\{-\left(\left.\sigma_{11}^{I I}\right|_{t=t_{B}}\right)\left(t_{0} / t_{B}\right) \pm \sqrt{\left[\left(\left.\sigma_{11}^{I I}\right|_{t=t_{B}}\right)\left(t_{0} / t_{B}\right)\right]^{2}+4 \mu_{B}\left(\mu_{1}+2 \mu_{2}+\mu_{B}\right)}\right\} /\left(2 \mu_{B}\right)$,

where (6.20) is also taken into consideration. Real solutions of (6.28) are possible only if the appearing material moduli are such that

$\mu_{B}\left(\mu_{1}+2 \mu_{2}+\mu_{B}\right) \geq-\left[\left(\left.\sigma_{11}^{I I}\right|_{t=t_{B}}\right)\left(t_{0} / t_{B}\right) / 2\right]^{2}$.

This inequality is thus perceived as a prerequisite condition in determining the value of $\mu_{B}$.

If, on the other hand, a set of values is provided for all material moduli appearing in (B.3), then this inequality is regarded as a restriction on the length of the time interval $\left[t_{A}, \hat{t}_{F}\right)$ that work-hardening deformation can take place $\left(t_{A}<t_{B}<\hat{t}_{F}\right)$. The final instant of that interval, $\hat{t}_{F}$, is generally different to its fracture counterpart, $t_{F}$, mentioned in the last paragraph of Section 5. If $t_{F}<\hat{t}_{F}$, then $\hat{t}_{F}$ exerts no influence in the outlined analysis. Otherwise, $\hat{t}_{F}$ is generally reached before fracture takes place, and is thus perceived as the last instant that the dilatation pattern (2.1) is attainable within the work-hardening regime. In such a case, $\hat{t}_{F}$ should necessarily be regarded as a point of material instability.

Provided that the set of values of the involved material moduli satisfies (B.3), admissible values of

the length parameter $\hat{L}_{\hat{0}}$ can be sought only among the positive real solutions of (6.28). Recall in this context that, in the present case of interest, the external loading is considered tensile and, therefore, $\sigma_{11}>0$. If follows that if the left hand side of (B.3) is positive, then only the positive square root appearing in (B.2) can provide an admissible value of $\hat{L}_{\hat{0}}$. If, on the other hand, $\mu_{B}>0$ and (B.3) is satisfied while its left hand side is negative, then (B.2) provides only inadmissible values of $\hat{L}_{\hat{0}}$. However, if $\mu_{B}<0$, then there should be cases in which (B.2) provides two admissible values of $\hat{L}_{\hat{0}}$.

\section{References}

[1] R.S. Rivlin, Large elastic deformations of isotropic materials. II. Some uniqueness theorems for pure homogeneous deformation, Phil. Trans. Roy. Soc. London A 240 (1948) 491-508.

[2] R.W. Ogden, On non-uniqueness in the traction boundary-value problem for a compressible elastic solid, Q. Appl. Math. 42 (1984) 337-344.

[3] R.S. Rivlin, M.F. Beatty, Dead loading of a unit cube of compressible isotropic elastic material, ZAMP 54 (2003) 954-963.

[4] D.M. Haughton, A comparison of stability and bifurcation criteria for a compressible elastic cube, $J$. Eng. Math. 53 (2005) 79-98.

[5] K.P. Soldatos, On the stability of a compressible Rivlin's cube made of transversely isotropic material. IMA J. Appl. Math. 71 (2006) 332-353.

[6] K.P. Soldatos, On the stability and non-uniqueness of the dilatation states of a compressible isotropic cube subjected to dead loading. Int. J. Non-Lin. Mech. 42 (2007) 271-282.

[7] A.M. Tarantino, Homogeneous equilibrium configurations of a hyperelastic compressible cube under equitriaxial dead-load tractions, J. Elast. 92 (2008) 227-254. 
[8] J.G. Lloyd, J. Sivaloganathan, On the relative energies of the Rivlin and cavitation instabilities for compressible materials, Math. Mech. Solids 17 (2011) 338-350.

[9] A.E. Green, P.M. Naghdi, General theory of an elastic-plastic Continuum, Arch. Rational Mech. Anal. 18 (1965) 251-281.

[10] R. Hill, The Mathematical Theory of Plasticity. Oxford, Clarendon Press, 1950.

[11] Malvern, L.E., Introduction to the Mechanics of a Continuous Medium. Prentice Hall, Englewood Cliffs, N.J., 1969.

[12] L.M. Kachanov, Foundations of the Theory of Plasticity. North-Holland Publ., Amsterdam, 1971.

[13] R. Rivlin, The solution of problems in second order elasticity theory, Arch. Rational Mech. Anal. 2 (1953) 53-81.

[14] R. Rivlin, Large elastic deformations, in Rheology, Theory and Applications 1, L. Eirich, ed., 351385, Academic Press, New York ,1956.

[15] A.J.M Spencer, A nonlinear viscoplasticity theory for transversely isotropic materials. In "Proc. $4^{\text {th }}$ Int. Conf. Nonlin. Mech (ICNM-IV), Sanghai, (Aug. 2002), pp.149-154.

[16] A.J.M Spencer, Deformations of Fibre-reinforced Materials. Clarendon Press, Oxford, 1972.

[17] Q.-S. Zheng, Theory of representations for tensor functions. Appl. Mech. Rev. 47 (1994) 554-587. 


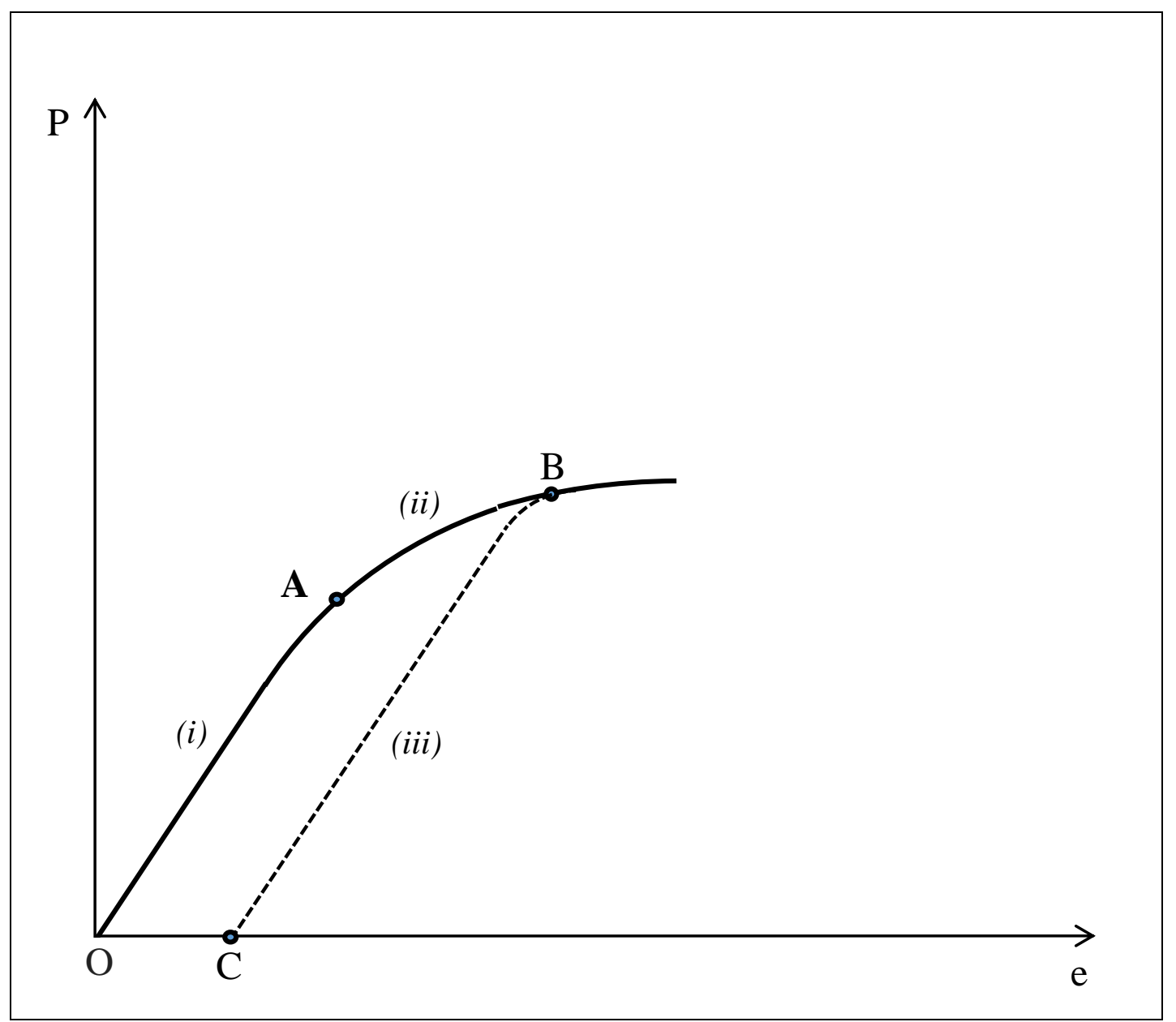

Figure 1: Idealisation of elastic-plastic behaviour of metals under negligible hysteresis and simple loading/unloading conditions; $P$ and $e$ represent external loading and strain, respectively. 


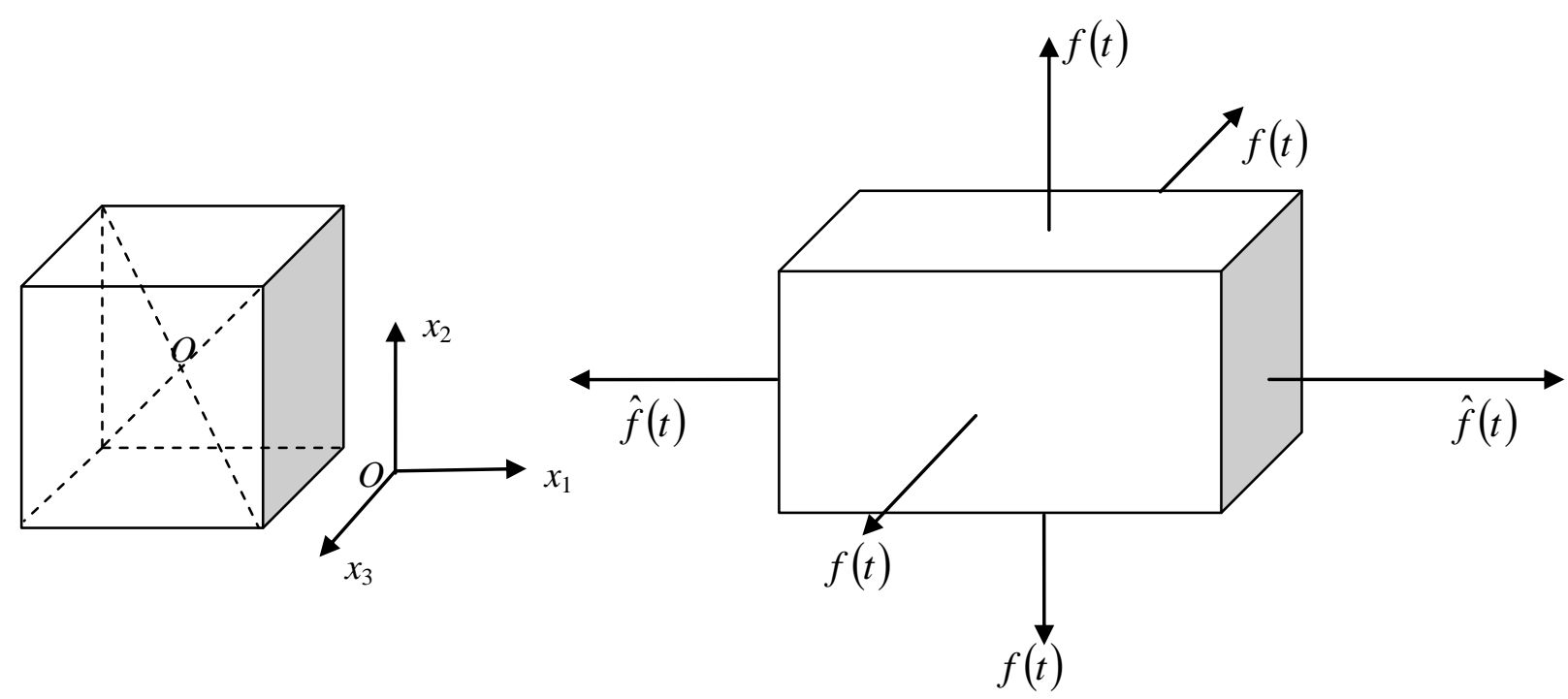

(a) $t=t_{0}$

(b) $t>t_{0}$

FIGURE 2: (a) Undeformed unit cube with edges parallel to the axes of the depicted Cartesian co-ordinate system; (b) Deformed cuboid configuration due to action of the externally applied pairs of tensile forces $\hat{f}>f>0$ (the magnitude of these forces and the dimensions of the resulting cuboid are determined in the text for all three deformations stages (i), (ii) and (iii) implied in Figure 1). 